\title{
SEMI-LOCALIZATION OF A ONE POINTED KAN COMPLEX
}

\author{
Phillip B. Thurber
}

This work was motivated by the goal of removing the hypothesis of simple connectedness from the rational homotopy theory of D. Sullivan. To a simply connected space $X$ is associated it's rational localization $\phi: \mathbf{X} \rightarrow \mathbf{X}_{0}$, and to the differential graded algebra $\mathbf{A}(X)$ of rational polynomial forms on $\mathbf{X}$ it's Sullivan minimal model $\psi: \mathbf{M} \rightarrow \mathbf{A}(\mathbf{X})$. It is shown that the minimal model $M$ is dual to the Postnikov tower of $X_{0}$. Thus $M$ determines the rational homotopy type of $\mathrm{X}$.

In the present paper we have eliminated the simply connected hypothesis from the first part of the theory. Working in the category of semi-simplicial complexes, we show that if $\mathrm{X}$ is a one pointed Kan complex, and $\mathrm{P}$ is a family of prime integers, there exists a semi-P-localization $f: \mathbf{X} \rightarrow \mathbf{X}_{p}$ such that $f_{*}: \pi_{1}(\mathbf{X}) \rightarrow \pi_{1}\left(\mathbf{X}_{p}\right)$ is an isomorphism and $f_{*}: \pi_{k}(\mathbf{X}) \rightarrow \pi_{k}\left(\mathbf{X}_{p}\right)$ is $\mathbf{P}$-localization of abelian groups, $k \geq 2$. Semi- P-localization is also characterized by a universal mapping property, and the fact that $f$ induces isomorphisms on twisted coefficient cohomology whenever the coefficients are in a $\mathbf{Z}_{(P)}$-module.

\section{Preliminaries.}

We use semi-simplicial homotopy theory, and notation for the most part as given in [8].

Definition 1.1. A semi-simplicial complex $\mathbf{K}$ is a sequence of sets $\left\{K_{n}\right\}_{n \geq 0}$ where $K_{n}$ is called the set of n-simplices of $\mathbf{K}$, together with functions $\partial_{i}$ : $K_{n} \rightarrow K_{n-1}$ and $s_{j}: K_{n} \rightarrow K_{n+1}, 0 \leq i, j \leq n$. If $\sigma \in K_{n}$ then $\partial_{i} \sigma$ is called the $i^{\text {th }}$-face and $s_{j} \sigma$ is called the $j^{\text {th }}$-degeneracy of $\sigma$. The functions $\partial_{i}$ and $s_{j}$ are required to satisfy the following relations:

$$
\begin{aligned}
& \partial_{i} \partial_{j}=\partial_{j-1} \partial_{i}, \quad i<j ; \\
& s_{i} s_{j}=s_{j+1} s_{i}, \quad i \leq j ; \\
& \partial_{i} s_{j}= \begin{cases}s_{j-1} \partial_{i} & \text { if } i<j ; \\
\text { identity } & \text { if } i=j, j+1 \\
s_{j} \partial_{i-1} & \text { if } i>j+1 .\end{cases}
\end{aligned}
$$


A semi-simplicial map $f: K \rightarrow L$ is a sequence of functions $f_{n}: K_{n} \rightarrow L_{n}$ such that

$$
\begin{array}{ll}
\partial_{i} f_{n}=f_{n-1} \partial_{i}, & 0 \leq i \leq n \\
s_{j} f_{n}=f_{n+1} s_{j}, & 0 \leq j \leq n .
\end{array}
$$

Definition 1.2. A semi-simplicial complex $\mathbf{K}$ is said to be Kan if for every pair of integers $(\mathrm{k}, \mathrm{n})$ with $0 \leq k \leq n$ and for every $\mathrm{n}(\mathrm{n}-1)$-simplices

$$
\sigma_{0}, \ldots, \sigma_{k-1}, \sigma_{k+1}, \ldots, \sigma_{n} \in K_{n-1}
$$

such that $\partial_{i} \sigma_{j}=\partial_{j-1} \sigma_{i}$ whenever $i<j$ and $i \neq k \neq j$, there exists an n-simplex $\sigma \in K_{n}$ such that $\partial_{i} \sigma=\sigma_{i}$ for all $i \neq k$.

Kan complexes play a role in Semi-simplicial homotopy theory that is analogous to that played by C.W. complexes in topological homotopy theory.

Definition 1.3. For $n \geq 0$ we define $\Delta[n]$ the standard semi-simplicial $n$-simplex as follows. A q-simplex of $\Delta[n]$ is a sequence $\left(a_{0}, a_{1}, \ldots, a_{q}\right)$ of integers $a_{i}$ such that

$$
0 \leq a_{0} \leq a_{1} \leq \ldots \leq a_{q} \leq n
$$

Face and degeneracy operators are defined by

$$
\begin{aligned}
& \partial_{i}\left(a_{0}, \ldots, a_{q}\right)=\left(a_{0}, \ldots, a_{i-1}, a_{i+1}, \ldots, a_{q}\right) ; \\
& s_{j}\left(a_{0}, \ldots, a_{q}\right)=\left(a_{0}, \ldots, a_{i}, a_{i}, \ldots, a_{q}\right) .
\end{aligned}
$$

We let $\sigma_{n}$ denote the unique non-degenerate n-simplex $(0,1, \ldots, \mathrm{n}) . \sigma_{n}$ generates $\Delta[n]$ in the sense that any simplex of $\Delta[n]$ can be obtained from $\sigma_{n}$ by applying a suitable sequence of face and degeneracy opperators.

$\Delta[1]$ will also be denoted by $\mathbf{I}$.

If $\mathbf{K}$ is a semi-simplicial complex, and $k \in K_{q}$ is a $q$-simplex, then there is a unique simplicial map $\bar{k}: \Delta[q] \rightarrow \mathbf{K}$ such that $\bar{k}\left(\sigma_{q}\right)=k$.

Definition 1.4. We define the total singular complex of a topological space $\mathbf{X}$ as follows. For $n \geq 0$ let $\Delta_{n}=\left\{\left(t_{0}, \ldots, t_{n}\right) \in \mathbf{R}^{n+1} \mid \quad 0 \leq t_{i} \leq\right.$ $\left.1, \Sigma t_{i}=1\right\}$. Define $e_{i}: \Delta_{n-1} \rightarrow \Delta_{n}$ by $e_{i}\left(t_{0}, \ldots, e_{n}\right)=\left(t_{0}, \ldots, t_{i-1}, 0, t_{i}, \ldots, t_{n}\right)$, $0 \leq i \leq n$. Define $f_{j}: \Delta_{n+1} \rightarrow \Delta_{n}$ by $f_{j}\left(t_{0}, \ldots, t_{n+1}\right)=\left(t_{0}, \ldots, t_{j-1}, t_{j}+\right.$ $\left.t_{j+1}, t_{j+2}, \ldots, t_{n+1}\right), 0 \leq j \leq n$.

If $\mathbf{X}$ is a topological space, a singular $\mathrm{n}$-simplex in $\mathbf{X}$ is a continuous map $\sigma: \Delta_{n} \rightarrow \mathbf{X}$. We define a semi-simplicial complex $\mathbf{S}(\mathbf{X})$, called the total 
singular complex of $\mathbf{X}$, by letting $S_{n}(\mathbf{X})$ be the set of all singular n-simplices in $\mathbf{X} . \partial_{i}: S_{n}(\mathbf{X}) \rightarrow S_{n-1}(\mathbf{X})$ and $s_{j}: S_{n}(\mathbf{X}) \rightarrow S_{n+1}(\mathbf{X})$ are defined by $\partial_{i} \sigma=\sigma \circ e_{i}, s_{j} \sigma=\sigma \circ f_{j}, \quad 0 \leq i, j \leq n$. If $f: \mathbf{X} \rightarrow \mathbf{Y}$ is a continuous map, $f$ induces the semi-simplicial map $\mathbf{S}(f): \mathbf{S}(\mathbf{X}) \rightarrow \mathbf{S}(\mathbf{Y})$ defined by $\mathbf{S}(f)(\sigma)=f \circ \sigma$. It is straight forward to show that $\mathbf{S}(\mathbf{X})$ is a Kan complex, and it is clear that $\mathbf{S}$ thus defined is a covariant functor from the category of topological spaces to the category of semi-simplicial complexes.

Definition 1.5. A simplicial group is a semi-simplicial complex $\mathbf{G}$ such that:

(i) The set $G_{n}$ of n-simplices is a group, $n \geq 0$;

(ii) The face and degeneracy operators $\partial_{i}: G_{n} \rightarrow G_{n-1}$, and $s_{j}: G_{n} \rightarrow$ $G_{n+1}$ are group homomorphisms, $0 \leq i, j \leq n$.

A simplicial group is a Kan complex, see [8, 17.1].

Definition 1.6. A simplicial group $\mathbf{G}$ is said to operate from the right on a semi-simplicial complex $\mathbf{E}$ if there is a semi-simplicial map $\phi: \mathbf{E} \times \mathbf{G} \rightarrow \mathbf{E}$ such that:

$$
\begin{aligned}
\phi\left(\sigma, e_{q}\right) & =\sigma ; \\
\phi\left(\sigma, g_{1} g_{2}\right) & =\left(\phi\left(\sigma, g_{1}\right), g_{2}\right) .
\end{aligned}
$$

We will denote $\phi(\sigma, g)$ by $\sigma g$. A left operation is defined similarly.

$\mathbf{G}$ is said to operate principally on $\mathbf{E}$ if whenever $\sigma g=\sigma$ for some $\sigma \in E_{q}$ we must have $g=e_{q}$. Notice that $\mathbf{G}$ operates principally on itself from both the left and the right. If $\mathbf{G}$ operates principally on $\mathbf{E}$, then we define a quotient complex $\mathbf{B}$ of $\mathbf{E}$ by identifying $\sigma$ and $\sigma g$ for all $\sigma \in E_{q}$, and $g \in G_{q}$. The natural map $p: \mathbf{E} \rightarrow \mathbf{B}$ is called a principal fibration with base $\mathbf{B}$ and structure group $\mathbf{G}$.

Definition 1.7. Let $\mathbf{F}$ and $\mathbf{B}$ be semi-simplicial complexes, and $\mathbf{G}$ a simplicial group which operates from the right on $\mathbf{F}$. A (right) twisted cartesian product, or TCP, with fiber $\mathbf{F}$, base $\mathbf{B}$, and group $\mathbf{G}$ is a semi-simplicial complex denoted by either $\mathbf{F} \times{ }^{\tau} \mathbf{B}$ or $\mathbf{E}(\tau)$, which satisfies $E(\tau)_{q}=F_{q} \times B_{q}$ and has face and degeneracy operators:

$$
\begin{aligned}
\partial_{0}(f, b) & =\left(\left(\partial_{0} f\right) \tau(b), \partial_{0} b\right) ; \\
\partial_{i}(f, b) & =\left(\partial_{i} f, \partial_{i} b\right), \quad i>0 ; \\
s_{i}(f, b) & =\left(s_{i} f, s_{i} b\right), \quad i \geq 0
\end{aligned}
$$

where $\tau: B_{q} \rightarrow G_{q-1}$ is called the twisting function. The requirement that $\mathbf{E}(\tau)$ be a semi-simplicial complex is equivalent to the following identities 
on $\tau$.

$$
\begin{aligned}
\tau\left(\partial_{1} b\right) & =\partial_{0} \tau(b) \tau\left(\partial_{0} b\right) ; \\
\tau\left(\partial_{i+1} b\right) & =\partial_{i} \tau(b), \quad i>0 ; \\
\tau\left(s_{0} b\right) & =e_{q}, \quad b \in B_{q} ; \\
\tau\left(s_{i+1} b\right) & =s_{i} \tau(b), \quad i \geq 0 .
\end{aligned}
$$

If $\mathbf{F}=\mathbf{G}$, then $\mathbf{E}(\tau)$ is called a principal TCP, or PTCP, as the projection of $\mathbf{E}(\tau)$ onto $\mathbf{B}$ is a princpal fibration.

\section{Eilenberg-MacLane Complexes.}

Definition 2.1. Let $\pi$ be an arbitrary group. Define a simplicial group $\mathbf{K}(\pi, 0)$ as follows:

$$
\begin{aligned}
K(\pi, 0)_{q} & =\pi, \quad q \geq 0 . \\
\partial_{1}=s_{j} & =i d .: \pi \rightarrow \pi, \quad 0 \leq i, j \leq q .
\end{aligned}
$$

$\mathbf{K}(\pi, 0)$ is a minimal complex of homotopy type $(\pi, 0)$.

Definition 2.2. $\quad$ Let $\mathbf{X}$ be a Kan complex with exactly one 0-simplex $\phi$. Let $\pi=\pi_{1}(\mathbf{X}, \phi)$. Define a graded function $\tau: X_{q} \rightarrow K(\pi, 0)_{q-1}$ by $\tau(x)=\left[\partial_{2} \ldots \partial_{q} x\right] \in \pi$. The function $\tau$ satisfies the following identities:

$$
\begin{aligned}
\tau\left(\partial_{1} x\right) & =\partial_{0} \tau(x) \tau\left(\partial_{0} x\right) ; \\
\tau\left(\partial_{i+1} x\right) & =\partial_{1} \tau(x), \quad i \geq 1 ; \\
\tau\left(s_{0} x\right) & =1 ; \\
\tau\left(s_{i+1} x\right) & =s_{i} \tau(x) .
\end{aligned}
$$

It follows that $\tau$ is a (right) twisting function.

Definition 2.3. Denote the $\operatorname{PTCP} \mathbf{K}(\pi, 0) \times{ }^{\tau} \mathbf{X}$ by $\widetilde{\mathbf{X}}$. $\widetilde{\mathbf{X}}$ is the universal covering complex of $\mathbf{X}$.

Definition 2.4. Let $\pi$ be an arbitrary group. define a semi-simplicial 
complex $\overline{\mathbf{W}}(\pi)$ as follows.

$$
\begin{aligned}
& \bar{W}(\pi)_{0}=(1) \\
& \bar{W}(\pi)_{q}=\underbrace{\pi \times \ldots \times \pi}_{q-\text { factors }}, \quad q>0 .
\end{aligned}
$$

(ii)

$$
\begin{aligned}
& \partial_{0}\left(x_{1}, \ldots, x_{q}\right)=\left(x_{2}, \ldots, x_{q}\right) \\
& \partial_{q}\left(x_{1}, \ldots, x_{q}\right)=\left(x_{1}, \ldots, x_{q-1}\right) \\
& \partial_{i}\left(x_{1}, \ldots, x_{q}\right)=\left(x_{1}, \ldots, x_{i-1}, x_{i} x_{i+1}, x_{i+2}, \ldots, x_{q}\right), \quad 1 \leq i \leq q-1,
\end{aligned}
$$

$$
s_{i}\left(x_{1}, \ldots, x_{q}\right)=\left(x_{1}, \ldots, x_{i}, 1, x_{i+1}, \ldots, x_{q}\right), \quad 0 \leq i \leq q .
$$

$\overline{\mathbf{W}}(\pi)$ is a minimal complex of homotopy type $(\pi, 1)$. Notice that $\partial_{2} \ldots \partial_{q}\left(x_{1}, \ldots, x_{q}\right)=\left(x_{1}\right)$, so the twisting function $\tau: \bar{W}(\pi)_{q} \rightarrow K(\pi, 0)_{q-1}$ of $(2.2)$ is given by $\tau\left(x_{1}, \ldots, x_{q}\right)=x_{1} \in \pi$.

Definition 2.5. Let $\mathbf{W}(\pi)$ denote the PTCP $\mathbf{K}(\pi, 0) \times^{\tau} \overline{\mathbf{W}}(\pi)$. Then $\mathbf{W}(\pi)$ is the universal covering complex of $\mathbf{W}(\pi)$. It follows that $\mathbf{W}(\pi)$ is contractible.

Definition 2.6. Let $G$ be an abelian group, $\Delta[q]$ be the standard semisimplicial q-simplex, and $n \geq 2$. Define a simplicial abelian group $\mathbf{C}(G, n+$ $1)$ as follows. Let $\mathbf{C}(G, n+1)_{q}=C^{n}(\Delta[q] ; G)$ the group of normalized n-cochains on $\Delta[q]$ with coefficients in $G$. An element of $\mathbf{C}(G, n+1)_{q}$ is determined by a function $\mu: \Delta[q]_{n} \rightarrow G$ such that $\mu(\alpha)=0$ whenever $\alpha$ is a degenerate n-simplex of $\Delta[q]$.

If $\mu \in C^{n}(\Delta[q] ; G)$ then $\partial_{i} \mu \in C^{n}(\Delta[q-1], G)$ and $s_{j} \mu \in C^{n}(\Delta[q+1] ; G)$ are defined by $\partial_{i} \mu=\mu \circ \delta_{i *}$ and $s_{j} \mu=\mu \circ \varsigma_{j_{*}}$ where $\delta_{i}: \Delta[q-1] \rightarrow \Delta[q]$ and $\varsigma_{j}: \Delta[q+1] \rightarrow \Delta[q]$ are determined by $\delta_{i}\left(\sigma_{q-1}\right)=\partial_{i} \sigma_{q}$, and $\varsigma_{j}\left(\sigma_{q+1}\right)=s_{j} \sigma_{q}$.

Define a simplicial group homomorphism $\delta: \mathbf{C}(G, n+1) \rightarrow \mathbf{C}(G, n+2)$ as follows. If $\mu \in C^{n}(\Delta[q] ; G)$ then $\delta \mu \in C^{n+1}(\Delta[q] ; G)$ is the usual coboundary. Now define $\mathbf{K}(G, n)=\operatorname{ker} \delta$. Then $\mathbf{K}(G, n)$ is a simplicial abelian group with $K(G, n)_{q}=Z^{n}(\Delta[q] ; G)$ the group of normalized n-cocycles on $\Delta[q]$ with coefficients in $\mathrm{G}$.

$H^{n}(\Delta[q] ; G)=0$ therefore $Z^{n+1}(\Delta[q] ; G)=\delta\left(C^{n}(\Delta[q] ; G)\right)$. Thus $\delta$ maps $\mathbf{C}(G, n+1)$ onto $\mathbf{K}(G, n+1)$ with kernel $\mathbf{K}(G, n) . \mathbf{K}(G, n)$ operates principally on $\mathbf{C}(G, n+1)$ by ordinary addition of cochains. It follows that $\delta: \mathbf{C}(G, n+1) \rightarrow \mathbf{K}(G, n+1)$ is a principal fibration with group $\mathbf{K}(G, n)$. $\mathbf{K}(G, n)$ is a minimal complex of homotopy type $(G, n)$ and $\mathbf{C}(G, n+1)$ is contractible. 
Definition 2.8. The correspondence $x \mapsto x\left(\sigma_{n}\right)$ between $C(G, n+1)_{n}$ and $G$ determines an $n$-cochain on $\mathbf{C}(G, n+1)$ with coefficients in $G$. Denote this cochain by $u_{n} \in C^{n}(\mathbf{C}(G, n+1) ; G) . u_{n}$ is called the fundamental cochain of $\mathbf{C}(G, n+1)$. Note that if $\mu \in C^{n}(\Delta[n+1] ; G)$ then $\left(\delta u_{n}\right)(\mu)=u_{n+1}(\delta \mu)$. It follows that $u_{n}$ restricted to $\mathbf{K}(G, n)$ is a cocycle. We denote this cocycle by $\omega_{n}$ and call it the fundamental cocycle of $\mathbf{K}(G, n)$. It's cohomology class is denoted by $\iota_{n}$.

Lemma 2.9. Let $\mathbf{K}$ be a semi-simplicial complex. Define

$$
\phi: \operatorname{Hom}_{\mathcal{S}}(\mathbf{K}, \mathbf{C}(G, n+1)) \rightarrow C^{n}(\mathbf{K} ; G)
$$

by $\phi(f)=f^{*}(u)$. Then $\phi$ is an isomorhism of groups with inverse $\psi$ defined by $\psi(\gamma)(\sigma)=\bar{\sigma}^{*}(\gamma)$ for $\gamma \in C^{n}(\mathbf{K} ; G), \sigma \in K_{n}$.

Proof. See $[8,24.2]$.

$\phi$ and $\psi$ restrict to give isomorphisms between the cocycles $Z^{n}(\mathbf{K}, G)$ and the maps $\operatorname{Hom}_{\mathcal{S}}(\mathbf{K}, \mathbf{K}(G, n))$. Finally we have:

Theorem 2.10. Let $f, g \in \operatorname{Hom}_{\mathcal{S}}(\mathbf{K}, \mathbf{K}(G, n))$ then $f \cong g$ if and only if $\phi(f)$ is cohomologous to $\phi(g)$. Hence there is a one to one correspondence between the set of homotopy classes $[\mathbf{K}, \mathbf{K}(G, n)]$ and the set of cohomology classes $H^{n}(\mathbf{K} ; G)$.

Proof. See $[8,24.4]$.

Definition 2.11. Let $\pi$ be a group, $\mathbf{K}$ be a semi-simplicial complex. By a simplicial operation of $\pi$ on $\mathbf{K}$ we mean an operation of $\mathbf{K}(\pi, 0)$ on $\mathbf{K}$. Note that if $\pi$ operates simplicially on $\mathbf{K}$, then each $x \in \pi$ determines a simplicial automorphism $x: \mathbf{K} \rightarrow \mathbf{K}$.

If $\pi$ opperates simplicially on the complexes $\mathbf{K}$ and $\mathbf{L}$, a map $f: \mathbf{K} \rightarrow \mathbf{L}$ is called equivariant if $f(x \sigma)=x f(\sigma)$ whenever $x \in \pi$ and $\sigma \in \mathbf{K}$.

If $\pi$ operates on the abelian group $G$, and operates simplicially on $\mathbf{K}$, $\gamma \in C^{n}(\mathbf{K} ; G)$ is said to be equivariant if $\gamma(x \sigma)=x \gamma(\sigma)$, for all $\sigma \in K_{n}$.

Definition 2.12. Let $\pi$ be a group, $G$ be an abelian group. Suppose $\pi$ operates on $G$, then $\pi$ operates simplicially on $\mathbf{C}(G, n+1)$ as follows. Suppose $\mu \in C^{n}(\Delta[q] ; G), x \in \pi$, and $\alpha \in \Delta[q]_{n}$, then $(x \mu)(\alpha)=x(\mu(\alpha)) \in$ $G$.

It should be noted that equivariant maps correspond to equivariant cochains in (2.9), and equivariant homotopy classes correspond to equivariant cohomology classes in (2.10). 


\section{3. $\mathbf{L}_{\pi}(G, n)$ 's and the classification Theorem.}

Definition 3.1. Let $\pi$ be a group. A $\pi$-module is a pair $(G, \phi)$ where $G$ is an abelian group, and $\phi: \pi \rightarrow \operatorname{Aut}(G)$ is a group homomorphism defining $\pi$ as a group of operators on $G$. A map $f:(G, \phi) \rightarrow\left(G^{\prime}, \phi^{\prime}\right)$ of $\pi$-modules is a group homomorphism $f: G \rightarrow G^{\prime}$ such that for all $x \in \pi, g \in G$, we have

$$
f(\phi(x)(g))=\phi^{\prime}(x)(f(g)) .
$$

If $(G, \phi)$ is a $\pi$-module, then $\phi$ induces simplicial operations of $\pi$ on $\mathbf{C}(G, n+$ $1)$ and $\mathbf{K}(G, n)$ as in (2.12).

Note that $\pi$ acts simplicially on $\mathbf{W}(\pi)$ by $x\left(x_{0}, x_{1}, \ldots, x_{q}\right)=\left(x x_{0}, \ldots, x_{q}\right)$.

Definition 3.2. In [4] a semi-simplicial complex $\mathbf{L}_{\pi}(G, n)$ is defined by

$$
\mathbf{L}_{\pi}(G, n)=\frac{\mathbf{K}(G, n) \times \mathbf{W}(\pi)}{\pi} .
$$

Where we quotient out by the diagonal operation induced by the left operations of $\pi$ on $\mathbf{K}(G, n)$ and $\mathbf{W}(\pi)$. If $f:(G, \phi) \rightarrow\left(G^{\prime}, \phi^{\prime}\right)$ is a map of $\pi$-modules then $f$ induces an equivariant map $f_{*}: \mathbf{K}(G, n) \rightarrow \mathbf{K}\left(G^{\prime}, n\right)$ by $f_{*}(\mu)=f \circ \mu, \mu \in Z^{n}(\Delta[q] ; G)$, and therefore a map $f_{*}: \mathbf{L}_{\pi}(G, n) \rightarrow$ $\mathbf{L}_{\pi}\left(G^{\prime}, n\right)$.

Let $\tau: \bar{W}(\pi)_{q} \rightarrow K(\pi, 0)_{q-1}$ be the twisting function (2.2). $\pi$ operates simplicially from the right on $\mathbf{K}(G, n)$ by $\mu x=\phi\left(x^{-1}\right) \circ \mu, x \in \pi$, $\mu \in Z^{n}(\Delta[q] ; G)$. Thus we may form the TCP $\mathbf{K}(G, n) \times^{\tau} \overline{\mathbf{W}}(\pi)$. Again if $f:(G, \phi) \rightarrow\left(G^{\prime}, \phi^{\prime}\right)$ is a map of $\pi$ - modules then $f$ induces a map $f_{*}: \mathbf{K}(G, n) \times{ }^{\tau} \overline{\mathbf{W}}(\pi) \rightarrow \mathbf{K}\left(G^{\prime}, n\right) \times{ }^{\tau} \overline{\mathbf{W}}(\pi)$.

Definition 3.3. Define maps $\psi: \mathbf{L}_{\pi}(G, n) \rightarrow \mathbf{K}(G, n) \times{ }^{\tau} \overline{\mathbf{W}}(\pi)$ and $\phi: \mathbf{K}(G, n) \rightarrow \mathbf{L}_{\pi}(G, n)$ by:

$$
\begin{aligned}
\psi\left[\mu,\left(x_{0}, x_{1}, \ldots, x_{q}\right)\right] & =\left(\mu x_{0},\left(x_{1}, \ldots, x_{q}\right)\right) ; \\
\phi\left(\mu,\left(x_{1}, \ldots, x_{q}\right)\right) & =\left[\mu,\left(1, x_{1}, \ldots, x_{q}\right)\right] .
\end{aligned}
$$

Proposition 3.4. $\psi$ and $\phi$ are inverse isomorphisms.

Proof. This is a straightforward check.

We will hence forth use $\mathbf{L}_{\pi}(G, n)$ to denote $\mathbf{K}(G, n) \times^{\tau} \overline{\mathbf{W}}(\pi) . \mathbf{L}_{\pi}(G, n)$ is a minimal complex, with exactly one 0 -simplex. It's fundamental group is 
$\pi$ and it has exactly one other non-vanishing homotopy group in dimension $\mathrm{n}$, which is isomorphic to $G$. The action of $\pi_{1}\left(\mathbf{L}_{\pi}(G, n)\right)$ on $\pi_{n}\left(\mathbf{L}_{\pi}(G, n)\right)$ is given by the homomorphism $\phi: \pi \rightarrow \operatorname{Aut}(G)$ which defines $G$ as a $\pi$ module.

Let $\widetilde{\mathbf{L}}$ be the universal covering complex of $\mathbf{L}_{\pi}(G, n)$.

Definition 3.5. Define maps $\zeta: \widetilde{\mathbf{L}} \rightarrow \mathbf{K}(G, n) \times \mathbf{W}(\pi)$ and $\eta: \mathbf{K}(G, n) \times$ $\mathbf{W}(\pi) \rightarrow \widetilde{\mathbf{L}}$ by:

$$
\begin{aligned}
\zeta\left(x, \mu,\left(x_{1}, \ldots, x_{q}\right)\right) & =\left(\mu x^{-1},\left(x, x_{1}, \ldots, x_{q}\right)\right) ; \\
\eta\left(\mu,\left(x_{0}, x_{1}, \ldots, x_{q}\right)\right) & =\left(x_{0}, \mu x_{0},\left(x_{1}, \ldots, x_{q}\right)\right) .
\end{aligned}
$$

Proposition 3.6. $\quad \zeta$ and $\eta$ are natural, equivariant, and inverse isomorphisms.

Proof. This is a straightforward check.

Definition 3.7. $\quad$ Define a map $p: \mathbf{K}(G, n) \times \mathbf{W}(\pi) \rightarrow \mathbf{L}_{\pi}(G, n)$ by $p\left(\mu,\left(x_{0}, x_{1}, \ldots, x_{q}\right)\right)=\left(\mu x_{0},\left(x_{1}, \ldots, x_{q}\right)\right)$. Notice that $p \zeta: \widetilde{\mathbf{L}} \rightarrow \mathbf{L}_{\pi}(G, n)$ is the usual projection. Consequently we may consider $p: \mathbf{K}(G, n) \times$ $\mathbf{W}(\pi) \rightarrow \mathbf{L}_{\pi}(G, n)$ to be the universal covering.

If $\mathbf{X}$ is a one pointed semi-simplicial complex, with $\pi=\pi_{1}(\mathbf{X}), \quad p: \widetilde{\mathbf{X}} \rightarrow$ $\mathbf{X}$ the universal covering, $(G, \phi)$ a $\pi$-module, let $C^{*}\left(\mathbf{X} ; G_{\phi}\right)$ be the cochain complex on $\mathbf{X}$ with coefficients in $G$ twisted by $\phi$, and $C_{\mathrm{ev}}^{*}(\widetilde{\mathbf{X}} ; G)$ the cochain complex on $\widetilde{\mathbf{X}}$ of equivariant cochains. We have the following theorem due to S. Eilenberg, see [2, 25.2].

Theorem 3.8. Let $\mathbf{X}$ be a one pointed Kan complex, $\pi=\pi_{1}(\mathbf{X}), p$ : $\widetilde{\mathbf{X}} \rightarrow \mathbf{X}$ be the universal covering complex. Let $(G, \phi)$ be a $\pi$-module, and let $C_{\mathrm{ev}}^{*}(\widetilde{\mathbf{X}} ; G)$ be the cochain complex of equivariant cochains. Then there is an isomorphism

$$
H^{q}\left(\mathbf{X} ; G_{\phi}\right) \cong H_{\mathrm{ev}}^{q}(\widetilde{\mathbf{X}} ; G)
$$

for all $q$, which is natural with respect to mappings of complexes with basepoint.

Proof. We define a natural cochain map $p_{\mathrm{ev}}^{*}: C^{*}\left(\mathbf{X} ; G_{\phi}\right) \rightarrow C_{\mathrm{ev}}^{*}(\widetilde{\mathbf{X}} ; G)$ as follows. Note that $\widetilde{X}_{0}=\pi$. Define $\gamma: \widetilde{X}_{q} \rightarrow \pi$ by $\gamma(T)=\partial_{1} \partial_{2} \ldots \partial_{q} T \in \pi$. For $T \in \widetilde{X}_{q}$, and $\mu \in C^{q}\left(\mathbf{X} ; G_{\phi}\right)$ define $p_{\mathrm{ev}}^{*}(\mu)(T)=\gamma(T) \mu(p(T))$. Now define $p_{\mathrm{ev}}^{*-1}: C_{\mathrm{ev}}^{*}(\widetilde{\mathbf{X}} ; G) \rightarrow C^{*}\left(\mathbf{X} ; G_{\phi}\right)$ by $p_{\mathrm{ev}}^{*-1}(h)(x)=h(1, x)$ It is straightforward to check that $p_{\mathrm{ev}}^{*}$ and $p_{\mathrm{ev}}^{*}{ }^{-1}$ are inverse isomorphisms of cochain complexes. 
Definition 3.9. Let $(G, \phi)$ be a $\pi$-module. Notice that the fundamental cocycle $u_{n} \in C^{n}(\mathbf{K}(G, n) ; G)$ is equivariant. Let $\iota_{n} \in H_{\mathrm{ev}}^{n}(\mathbf{K}(G, n) ; G)$ be it's class. Now consider the diagram

$$
\mathbf{L}_{\pi}(G, n) \stackrel{p}{\longleftarrow} \mathbf{K}(G, n) \times \mathbf{W}(\pi) \stackrel{p_{1}}{\longrightarrow} \mathbf{K}(G, n)
$$

where $p$ is defined in (3.7) and $p_{1}$ is projection on the first factor. $p_{1}$ is an equivariant map so we have the induced diagram

$$
H^{n}\left(\mathbf{L}_{\pi}(G, n) ; G_{\phi}\right) \stackrel{p_{\mathrm{ev}}^{*}}{\cong} H_{\mathrm{ev}}^{n}(\mathbf{K}(G, n) \times \mathbf{W}(\pi) ; G) \stackrel{p_{1}{ }^{*}}{\longleftarrow} H^{n}(\mathbf{K}(G, n) ; G) .
$$

We define the fundamental class $\lambda_{n} \in H^{n}\left(\mathbf{L}_{\pi}(G, n) ; G_{\phi}\right)$ by $\lambda_{n}=$ $\left(p_{\mathrm{ev}}^{*}\right)^{-1} p_{1}^{*}\left(\iota_{n}\right)$. Notice that if $i: \mathbf{K}(G, n) \rightarrow \mathbf{L}_{\pi}(G, n)$ is inclusion of the fiber, then $i^{*} \lambda_{n}=\iota_{n}$.

Definition 3.10. Let $\mathbf{X}, \mathbf{Y}$ be one pointed complexes. Let $\alpha: \pi_{1}(\mathbf{X}) \rightarrow$ $\pi_{1}(\mathbf{Y})$ be a group homomorphism. Let $\pi_{\alpha}(\mathbf{X}, \mathbf{Y})$ be the set of homotopy classes of mappings which induce $\alpha$ on fundamental groups. We have the following theorem due to S. Gitler:

Theorem 3.11. Let $\mathbf{X}$ be a one pointed complex, and $\alpha: \pi_{1}(\mathbf{X}) \rightarrow \pi$ be a group homomorphism, then the function which to every $f \in \pi_{\alpha}\left(\mathbf{X}, \mathbf{L}_{\pi}(G, n)\right)$ assigns the class $f^{*} \lambda \in H^{n}\left(\mathbf{X} ; G_{\phi \alpha}\right)$ establishes a one-to-one correspondence between $\pi_{\alpha}\left(\mathbf{X}, \mathbf{L}_{\pi}(G, n)\right)$ and $H^{n}\left(\mathbf{X} ; G_{\phi \alpha}\right)$.

We will need the following result:

Lemma 3.12. Let $\mathbf{X}$ be a one pointed complex, and $\alpha: \pi_{1}(\mathbf{X}) \rightarrow \pi$ be a group homomorphism. Then there is a unique map $g_{\alpha}: \mathbf{X} \rightarrow \overline{\mathbf{W}}(\pi)$ inducing $\alpha$ on the fundamental groups.

Proof. (i) Uniqueness.

Suppose $f: \mathbf{X} \rightarrow \overline{\mathbf{W}}(\pi)$ induces $\alpha$ on fundamental groups. Since both $\mathbf{X}$ and $\overline{\mathbf{W}}(\pi)$ are one ponted we must have $f\left(x_{0}\right)=(1)$. Suppose $x \in X_{1}$, and let $\gamma=[x] \in \pi_{1}(\mathbf{X})$. Then $f(x)=\alpha(\gamma) \in \pi$ since $\overline{\mathbf{W}}(\pi)$ is a minimal complex. Now suppose $x \in X_{q}, q>1$. Let $\left(a_{1}, \ldots, a_{q}\right)=f(x) \in \bar{W}(\pi)_{q}$. Notice that $\left(a_{i}\right)=\partial_{0}^{i-1} \partial_{i+1}^{q-i}\left(a_{1}, \ldots, a_{q}\right), 1 \leq i \leq q$. Set $\gamma_{i}(x)=\left[\partial_{0}^{i-1} \partial_{i+1}^{q-i} x\right] \in \pi_{1}(\mathbf{X})$, $1 \leq i \leq q$. Then

$$
\begin{aligned}
\left(a_{i}\right) & =\partial_{0}^{i-1} \partial_{i+1}^{q-i} f(x) \\
& =f\left(\partial_{0}^{i-1} \partial_{i+1}^{q-i} x\right) \\
& =\alpha\left(\gamma_{i}(x)\right) .
\end{aligned}
$$

Hence $f(x)=\left(\alpha\left(\gamma_{1}(x)\right), \ldots, \alpha\left(\gamma_{q}(x)\right)\right)$. 
(ii) Existence.

It is straight forward to check that the function defined above is a simplicial map.

\section{The twisted coefficient serre spectral sequence.}

Definition 4.1. Let $p: \mathbf{E} \rightarrow \mathbf{B}$ be a Kan fibration, with fiber $\mathbf{F}=$ $p^{-1}\left(b_{0}\right)$. Let $(G, \phi)$ be a $\pi_{1}\left(\mathbf{B}, b_{0}\right)$ - module. We define a $\pi_{1}\left(\mathbf{B}, b_{0}\right)$ - module $\left(H^{q}(\mathbf{F} ; G), \tilde{\phi}\right)$ as follows. For each $\alpha \in \pi_{1}\left(\mathbf{B}, b_{0}\right)$ there is a homotopy class of automorphisms $\bar{\alpha}: \mathbf{F} \rightarrow \mathbf{F}$ such that $\overline{\alpha \beta}=\bar{\beta} \bar{\alpha}$. Define an automorphism $\tilde{\phi}(\alpha): H^{q}(\mathbf{F} ; G) \rightarrow H^{q}(\mathbf{F} ; G)$ by $\tilde{\phi}(\alpha)=\phi(\alpha)_{*} \bar{\alpha}^{*}=\bar{\alpha}^{*} \phi(\alpha)_{*}$.

The transgression $\tau: H^{n}(\mathbf{F} ; G) \rightarrow H^{n+1}\left(\mathbf{B} ; G_{\phi}\right)$ is defined by $\tau=j^{*} \bar{p}^{*-1} \delta$ where

$$
H^{n}(\mathbf{F} ; G) \stackrel{\delta}{\longrightarrow} H^{n+1}\left(\mathbf{E}, \mathbf{F} ; G_{\phi p_{*}}\right) \stackrel{\bar{p}^{*}}{\longleftarrow} H^{n+1}\left(\mathbf{B}, b_{0} ; G_{\phi}\right) \stackrel{j}{\cong} H^{n+1}\left(\mathbf{B} ; G_{\phi}\right) .
$$

The suspension is $\sigma=\tau^{-1}=\delta^{-1} \bar{p}^{*} j^{*-1}$. In general, $\tau$ and $\sigma$ are inverse additive relations.

Theorem 4.2. There is a natural first quadrant spectral sequence $\left\{E_{r}^{p, q}\right\}$ with differentials $d_{r}$ of bi-degree $(r, 1-r)$ and

$$
\begin{aligned}
& E_{1}^{p, q} \cong C^{p}\left(\mathbf{B} ; H^{q}(\mathbf{F} ; G)_{\tilde{\phi}}\right) \\
& E_{2}^{p, q} \cong H^{p}\left(\mathbf{B} ; H^{q}(\mathbf{F} ; G)_{\tilde{\phi}}\right),
\end{aligned}
$$

and $\left\{E_{\infty}^{p, q}\right\}_{p+q=n}$ gives the graded object associated to the induced filtration on $H^{n}\left(\mathbf{E} ; G_{\phi p_{*}}\right)$. Furthermore the additive relation:

$$
H^{n}(\mathbf{F} ; G)=E_{1}^{0, n} \stackrel{1-1}{\longleftarrow} E_{n+1}^{0, n} \stackrel{d_{n+1}}{\longrightarrow} E_{n+1}^{n+1,0} \stackrel{\text { onto }}{\longleftarrow} E_{2}^{n+1,0}=H^{n+1}\left(\mathbf{B} ; G_{\phi}\right)
$$

is the transgression. In particular

$$
\text { Def } \begin{aligned}
\tau \subset E_{2}^{n, 0} & =\left[H^{n}(\mathbf{F} ; G)\right]^{\tilde{\phi}} \\
& =\left\{x \in H^{n}(\mathbf{F} ; G) \mid \tilde{\phi}(\alpha) x=x \text { for all } \alpha \in \pi_{1}(\mathbf{B})\right\} .
\end{aligned}
$$

If $H^{p}\left(\mathbf{B} ; H^{q}(\mathbf{F} ; G)_{\tilde{\phi}}\right)=0$ for $0<q<n$, then $p^{*}: H^{i}\left(\mathbf{B} ; G_{\phi}\right) \rightarrow H^{i}\left(\mathbf{E} ; G_{\phi p_{*}}\right)$ is an isomorphism for all $i<n$, and the following is an exact sequence of qroups and group homomorphisms

$$
\begin{aligned}
0 \rightarrow H^{n}\left(\mathbf{B} ; G_{\phi}\right) & \stackrel{p^{*}}{\rightarrow} H^{n}\left(\mathbf{E} ; G_{\phi p_{*}}\right) \stackrel{i^{*}}{\rightarrow} H^{n}(\mathbf{F} ; G)^{\tilde{\phi}} \\
& \stackrel{\tau}{\rightarrow} H^{n+1}\left(\mathbf{B} ; G_{\phi}\right) \stackrel{p^{*}}{\rightarrow} H^{n+1}\left(\mathbf{E} ; G_{\phi p_{*}}\right),
\end{aligned}
$$


see $[9,1.1]$.

Definition 4.3. Let $(G, \phi)$ be a $\pi$-module. $\pi$ operates simplicially from the right on $\mathbf{C}(G, n+1)$ by $\mu x=\phi\left(x^{-1}\right) \circ \mu, \quad x \in \pi, \mu \in C^{n}(\Delta[q] ; G)$. Thus we can form the TCP $\mathbf{P}_{\pi}(G, n+1)=\mathbf{C}(G, n+1) \times{ }^{\tau} \overline{\mathbf{W}}(\pi)$.

The map $\delta: \mathbf{C}(G, n+1) \rightarrow \mathbf{K}(G, n+1)$ is equivariant, so it induces a map $p: \mathbf{P}_{\pi}(G, n+1) \rightarrow \mathbf{L}_{\pi}(G, n+1)$ by $p\left(\mu,\left(x_{1}, \ldots, x_{q}\right)\right)=\left(\delta \mu,\left(x_{1}, \ldots, x_{q}\right)\right) . p$ is a minimal Kan fibration with fiber $\mathbf{K}(G, n)$.

Notice that $\mathbf{K}(G, n)$ is a subcomplex of $\mathbf{C}(G, n+1)$, so $\mathbf{L}_{\pi}(G, n)$ is a subcomplex of $\mathbf{P}_{\pi}(G, n+1)$.

Lemma 4.4. Let $\iota_{n} \in H^{n}(\mathbf{K}(G, n) ; G)$ be the fundamental class of $\mathbf{K}(G, n)$, and let $\lambda_{n+1} \in H^{n+1}\left(\mathbf{L}_{\pi}(G, n+1) ; G_{\phi}\right)$ be the fundamental class of $\mathbf{L}_{\pi}(G, n+$ 1). If $\tau: H^{n}(\mathbf{K}(G, n) ; G) \rightarrow H^{n+1}\left(\mathbf{L}_{\pi}(G, n+1) ; G_{\phi}\right)$ is transgression for the Kan fibration $\mathbf{K}(G, n) \stackrel{i}{\rightarrow} \mathbf{P}_{\pi}(G, n+1) \stackrel{p}{\rightarrow} \mathbf{L}_{\pi}(G, n+1)$, then $\tau\left(\iota_{n}\right)=\lambda_{n+1}$.

Proof. Let $u_{q} \in C^{q}(\mathbf{C}(G, q+1) ; G)$ be the fundamental cochain, and let $\omega_{q}=i^{*} u_{q}$, then $\omega_{q}$ is the fundamental cocycle of $\mathbf{K}(G, q)$. Define $U_{n} \in$ $C^{n}\left(\mathbf{P}_{\pi}(G, n+1) ; G\right)$ by $U_{n}\left(\mu,\left(x_{1}, \ldots, x_{n}\right)\right)=u_{n}(\mu)$. Notice that $\delta U_{n}\left(\mu,\left(x_{1}, \ldots, x_{n+1}\right)\right)=\delta u_{n}(\mu)$. Thus $i^{*}\left(\delta U_{n}\right)=\delta \omega_{n}=0$ so $\delta U_{n} \in$ $C^{n+1}\left(\mathbf{P}_{\pi}(G, n+1), \mathbf{K}(G, n) ; G_{\phi}\right)$. It follows that if $\delta: H^{n}(\mathbf{K}(G, n) ; G) \rightarrow$ $H^{n+1}\left(\mathbf{P}_{\pi}(G, n+1), \mathbf{K}(G, n) ; G_{\phi}\right)$ is the connecting homomorphism then $\delta \iota_{n}=\left[\delta U_{n}\right]$.

We claim that $\bar{p}^{*} j^{*-1} \lambda_{n+1}=\left[\delta U_{n}\right]$.

Let $\Omega_{n+1} \in C^{n+1}\left(\mathbf{L}_{\pi}(G, n+1) ; G_{\phi}\right)$ be defined by $\Omega_{n+1}\left(\mu,\left(x_{1}, \ldots, x_{n+1}\right)\right)=$ $\omega_{n+1}(\mu)$. If $\rho: \mathbf{K}(G, n+1) \times \mathbf{W}(\pi) \rightarrow \mathbf{L}_{\pi}(G, n+1)$ is the universal covering complex, then $\rho_{\mathrm{ev}}^{*} \Omega_{n+1} \in C^{n+1}\left(\mathbf{L}_{\pi}(G, n+1) ; G_{\phi}\right)$ is given by

$$
\begin{aligned}
\rho_{\mathrm{ev}}^{*} \Omega_{n+1}\left(\mu,\left(x_{0}, x_{1}, \ldots, x_{n+1}\right)\right) & =x_{0} \Omega_{n+1}\left(\mu x_{0},\left(x_{1}, \ldots, x_{n+1}\right)\right) \\
& =x_{0} \omega_{n+1}\left(x_{0}^{-1} \mu\right) \\
& =\omega_{n+1}(\mu) \\
& =\left(p_{1}^{*} \omega_{n+1}\right)\left(\mu,\left(x_{0}, x_{1}, \ldots, x_{n+1}\right)\right) .
\end{aligned}
$$

Hence $\rho_{\mathrm{ev}}^{*} \Omega_{n+1}$ represents $p_{1}^{*}\left(\iota_{n+1}\right)$. It follows from the definition of $\lambda_{n+1}$ that $\Omega_{n+1}$ represents $\lambda_{n+1}$.

Notice that

$$
\begin{aligned}
p_{\mathrm{ev}}^{*} \Omega_{n+1}\left(\mu,\left(x_{1}, \ldots, x_{n+1}\right)\right) & =\Omega_{n+1}\left(\delta \mu,\left(x_{1}, \ldots, x_{n+1}\right)\right) \\
& =\omega_{n+1}(\delta \mu) \\
& =\delta u_{n}(\mu) \\
& =\delta U_{n}\left(\mu,\left(x_{1}, \ldots, x_{n+1}\right)\right) .
\end{aligned}
$$


Hence $\bar{p}^{*} j^{*-1} \lambda_{n+1}=\left[\delta U_{n}\right]$. Therefore $\lambda_{n+1} \in j^{*} \bar{p}^{*-1} \delta\left(\iota_{n}\right)=\tau\left(\iota_{n}\right)$. or equivalently $\iota_{n} \in \sigma\left(\lambda_{n+1}\right)$.

\section{Geometric realization and the Postnikov tower.}

This section follows McClendon [9], except that he worked in the category of C.W. complexes.

Definition 5.1. Let $\mathbf{D}$ be a semi-simplicial complex. A complex over $\mathbf{D}$ is a pair $(\mathbf{X}, \hat{x})$ where $\mathbf{X}$ is a semi-simplicial complex and $\hat{x}: \mathbf{X} \rightarrow \mathbf{D}$ is a semi-simplicial map. A map $f:(\mathbf{X}, \hat{x}) \rightarrow(\mathbf{Y}, \hat{y})$ over $\mathbf{D}$ is a semi-simplicial map $f: \mathbf{X} \rightarrow \mathbf{Y}$ such that $\hat{y} f=\hat{x}$. A $\mathbf{D}$-sectioned complex is a triple $(\mathbf{X}, \hat{x}, \check{x})$ such that $(\mathbf{X}, \hat{x})$ is a complex over $\mathbf{D}$, and $\check{x}: \mathbf{D} \rightarrow \mathbf{X}$ is a semi-simplicial map satisfying $\hat{x} \check{x}=1_{D}$. If $(\mathbf{X}, \check{x})$ is a complex over $\mathbf{D}$, then $\mathbf{X} \times \mathbf{I}$ is a complex over $\mathbf{D}$ via $\mathbf{X} \times \mathbf{I} \stackrel{p_{1}}{\rightarrow} \mathbf{X} \stackrel{\hat{x}}{\rightarrow} \mathbf{D}$.

A homotopy over $\mathbf{D}$ is a map $H: \mathbf{X} \times \mathbf{I} \rightarrow \mathbf{Y}$ over $\mathbf{D}$. $H \circ i_{0}$ and $H \circ i_{1}$ are homotopic over $\mathbf{D}$. Let $[\mathbf{X}, \mathbf{Y}]_{D}$ denote the set of homotopy classes of maps over $\mathbf{D}$. $\quad[\mathbf{X}, \mathbf{Y}]_{D}$ may be empty, but if $\mathbf{Y}$ is a $\mathbf{D}$-sectioned complex then $[\mathbf{X}, \mathbf{Y}]_{D}$ is non-empty and has the class $[\check{y} \hat{x}]_{D}$ as a distinguished element.

If $\mathbf{X}$ and $\mathbf{Y}$ are complexes over $\mathbf{D}$ define their product over $\mathbf{D}, \mathbf{X} \times_{D} \mathbf{Y}$, by

$$
\left(\mathbf{X} \times{ }_{D} \mathbf{Y}\right)_{q}=\left\{(x, y) \in X_{q} \times Y_{q} \mid \hat{x}(x)=\hat{y}(y)\right\} .
$$

$\mathbf{X} \times{ }_{D} \mathbf{Y}$ is again a complex over $\mathbf{D}$ via the map $(x, y) \mapsto \hat{x}(x)=\hat{y}(y) . \mathbf{A}$ Kan fibration over $\mathbf{D}$ is a Kan fibration $p: \mathbf{E} \rightarrow \mathbf{B}$ such that $p$ is a map over D. Suppose $\mathbf{X}$ is a complex over $\mathbf{D}, p: \mathbf{E} \rightarrow \mathbf{B}$ is a Kan fibration over $\mathbf{D}$, and $f: \mathbf{X} \rightarrow \mathbf{B}$ is a map over $\mathbf{D}$. Define the pull-back over $\mathbf{D}$ of $p$ by $f$ to be $q: \mathbf{E}^{f} \rightarrow \mathbf{X}$ where

$$
E_{n}^{f}=\left\{(e, x) \in\left(E \times_{D} X\right)_{n} \mid p(e)=f(x)\right\}
$$

with obvious face and degeneracy operators, and $q(e, x)=x$. Then $\mathbf{E}^{f}$ is a complex over $\mathbf{D}$ and $q$ is a Kan fibration over $\mathbf{D}$. Note that if $p$ is a minimal fibration then so is $q$.

Example 5.2. $\quad \mathbf{P}_{\pi}(G, n+1)$, and $\mathbf{L}_{\pi}(G, n+1)$ are $\overline{\mathbf{W}}(\pi)$-sectioned complexes via the maps $p_{2}$ and $s_{0}$ where

$$
\begin{aligned}
p_{2}\left(\mu,\left(x_{1}, \ldots, x_{q}\right)\right) & =\left(x_{1}, \ldots, x_{q}\right) ; \\
s_{0}\left(x_{1}, \ldots, x_{q}\right) & =\left(0,\left(x_{1}, \ldots, x_{q}\right)\right) .
\end{aligned}
$$

Furthermore $\mathbf{L}_{\pi}(G, n) \stackrel{i}{\rightarrow} \mathbf{P}_{\pi}(G, n+1) \stackrel{p}{\rightarrow} \mathbf{L}_{\pi}(G, n+1)$ is a sequence of maps of $\overline{\mathbf{W}}(\pi)$-sectioned complexes, and $p$ is a fibration over $\overline{\mathbf{W}}(\pi)$. 
Lemma 5.3. If $(\mathbf{X}, \hat{x})$ is a complex over $\overline{\mathbf{W}}(\pi)$ then $\left[\mathbf{X}, \mathbf{L}_{\pi}(G, n+1)\right]_{\overline{\mathbf{W}}(\pi)}$ has an abelian group structure with identity element $\left[s_{0} \hat{x}\right]$.

Proof. Let $f: \mathbf{X} \rightarrow \mathbf{L}_{\pi}(G, n+1)$ be a map over $\overline{\mathbf{W}}(\pi)$. Then $p_{2} f=\hat{x}$ implies $f(x)=\left(f_{1}(x), \hat{x}(x)\right)$ where $f_{1}$ is a graded function of degree $0, \mathbf{X} \rightarrow$ $\mathbf{K}(G, n+1)$, satisfying

$$
\begin{aligned}
& f_{1}\left(\partial_{0} x\right)=\partial_{0} f_{1}(x) \tau \hat{x}(x) \\
& f_{1}\left(\partial_{i} x\right)=\partial_{i} f_{1}(x), \quad i>0 ; \\
& f_{1}\left(s_{j} x\right)=s_{j} f_{1}(x), \quad j \geq 0 .
\end{aligned}
$$

If $f, g: \mathbf{X} \rightarrow \mathbf{L}_{\pi}(G, n+1)$ are maps over $\overline{\mathbf{W}}(\pi)$, define $f+g: \mathbf{X} \rightarrow$ $\mathbf{L}_{\pi}(G,+1)$ by $(f+g)(x)=\left(f_{1}(x)+g_{1}(x), \hat{x}(x)\right)$. It is clear that $f+g$ is again a map over $\overline{\mathbf{W}}(\pi)$. Also $\left(f+s_{0} \hat{x}\right)(x)=\left(f_{1}(x)+0, \hat{x}(x)\right)=f(x)$ and if $(-f)(x)=\left(-f_{1}(x), \hat{x}(x)\right)$ then $f+(-f)=s_{0} \hat{x}$.

Furthermore, suppose $F$ is a homotopy $f \cong f^{\prime}$ over $\overline{\mathbf{W}}(\pi)$ and $\mathrm{G}$ is a homotopy $g \cong g^{\prime}$ over $\overline{\mathbf{W}}(\pi)$. Then as maps $\mathbf{X} \times \mathbf{I} \rightarrow \mathbf{L}_{\pi}(G, n+1)$ we can form $F+G$ which is a homotopy $(f+g) \cong\left(f^{\prime}+g^{\prime}\right)$ over $\overline{\mathbf{W}}(\pi)$. Therefore the addition is defined on homotopy classes over $\overline{\mathbf{W}}(\pi)$.

Lemma 5.4. Suppose $(\mathbf{X}, \hat{x})$ is a one pointed complex over $\overline{\mathbf{W}}(\pi)$. If $\hat{x}$ induces the homomorphism $\quad \alpha: \pi_{1}(\mathbf{X}) \rightarrow \pi$, then

$$
\left[\mathbf{X}, \mathbf{L}_{\pi}(G, n+1)\right]_{\overline{\mathbf{W}}(\pi)} \cong \pi_{\alpha}\left(\mathbf{X}, \mathbf{L}_{\pi}(G, n+1)\right) .
$$

Proof. Let $f: \mathbf{X} \rightarrow \mathbf{L}_{\pi}(G, n+1)$ be a map over $\overline{\mathbf{W}}(\pi)$. Then $p_{2} f=\hat{x}$ implies $p_{2_{*}} f_{*}=\alpha$ on fundamental groups. But $p_{2_{*}}=i d .: \pi \rightarrow \pi$ therefore $f_{*}=\alpha$.

Conversely, if $f: \mathbf{X} \rightarrow \mathbf{L}_{\pi}(G, n+1)$ is an arbitrary semi-simplicial map inducing $\alpha$ on fundamental groups, then $\left(p_{2} f\right)_{*}=\alpha$. But by $(\mathbf{8 . 1 6})$ there is a unique map $\mathbf{X} \rightarrow \overline{\mathbf{W}}(\pi)$ inducing $\alpha$ on fundamental groups. Thus $p_{2} f=\hat{x}$.

A similar argument shows that if $H:\left(\mathbf{X} \times \mathbf{I}, x_{0} \times \mathbf{I}\right) \rightarrow\left(\mathbf{L}_{\pi}(G, n+1),(1)\right)$ is a homotopy $f \cong f^{\prime}\left(\right.$ rel $\left.x_{0}\right)$ of maps inducing $\alpha$ on fundamental groups, then $H$ is a homotopy over $\overline{\mathbf{W}}(\pi)$.

Now, let $\lambda_{n+1} \in H^{n+1}\left(\mathbf{L}_{\pi}(G, n+1) ; G_{\phi}\right)$ be the fundamental class, and let $\Omega_{n+1} \in C^{n+1}\left(\mathbf{L}_{\pi}(G, n+1) ; G_{\phi}\right)$ be it's representative as in (4.4). Recall that $\Omega_{n+1}\left(\mu,\left(x_{1}, \ldots, x_{n+1}\right)\right)=\omega_{n+1}(\mu)$. If $f, g: \mathbf{X} \rightarrow \mathbf{L}_{\pi}(G, n+1)$ over $\overline{\mathbf{W}}(\pi)$ then:

$$
\begin{aligned}
(f+g)^{*} \Omega_{n+1}(x) & =\omega_{n+1}\left(f_{1}(x)+g_{1}(x)\right) \\
& =\omega_{n+1}\left(f_{1}(x)\right)+\omega_{n+1}\left(g_{1}(x)\right) \\
& =f^{*} \Omega_{n+1}(x)+g^{*} \Omega_{n+1}(x) .
\end{aligned}
$$


It follows that $(f+g)^{*} \lambda_{n+1}=f^{*} \lambda_{n+1}+g^{*} \lambda_{n+1}$. Taking account of (3.11) and (5.4) we now have:

Theorem 5.5. Let $(\mathbf{X}, \hat{x})$ be a complex over $\overline{\mathbf{W}}(\pi)$. Then the function which assigns to each $f \in\left[\mathbf{X} ; \mathbf{L}_{\pi}(G, n+1)\right]_{\overline{\mathbf{W}}(\pi)}$ the class $f^{*} \lambda_{n+1} \in$ $H^{n+1}\left(\mathbf{L}_{\pi}(G, n+1) ; G_{\phi}\right)$ is a group isomorphism.

Lemma 5.6. If $(\mathbf{X}, \hat{x})$ is a complex over $\overline{\mathbf{W}}(\pi)$ where $\hat{x}$ is the trivial map, then

$$
\left[\mathbf{X}, \mathbf{L}_{\pi}(G, n)\right]_{\overline{\mathbf{W}}(\pi)} \cong[\mathbf{X}, \mathbf{K}(G, n)] .
$$

Proof. Since $\hat{x}$ is the trivial map, and $\tau\left(s_{0}^{q}(1)\right)=1, f: \mathbf{X} \rightarrow \mathbf{L}_{\pi}(G, n)$ is a map over $\overline{\mathbf{W}}(\pi)$ if and only if $f(x)=\left(f_{1}(x), s_{0}^{q}(1)\right)$, where $f_{1}: \mathbf{X} \rightarrow$ $\mathbf{K}(G, n)$ is a semi-simplicial map. Furthermore $H: \mathbf{X} \times \mathbf{I} \rightarrow \mathbf{L}_{\pi}(G, n)$ is a homotopy over $\overline{\mathbf{W}}(\pi)$ if and only if $H(x, \sigma)=\left(H_{1}(x, \sigma), s_{0}^{q}(1)\right)$ where $H_{1}: \mathbf{X} \rightarrow \mathbf{K}(G, n)$ is a semi-simplicial map.

Lemma 5.7. If $(\mathbf{X}, \hat{x})$ is as in (10.7) and $f: \mathbf{X} \rightarrow \mathbf{Y}$ is a map over $\overline{\mathbf{W}}(\pi)$, then

$$
f^{*}:\left[\mathbf{Y}, \mathbf{L}_{\pi}(G, n)\right]_{\overline{\mathbf{W}}(\pi)} \rightarrow[\mathbf{X}, \mathbf{K}(G, n)]
$$

is a group homomorphism.

Proof. Since $f$ is a map over $\overline{\mathbf{W}}(\pi)$ it follows that $\hat{y} f$ is trivial. If $g, h$ : $\mathbf{Y} \rightarrow \mathbf{L}_{\pi}(G, n)$ are maps over $\overline{\mathbf{W}}(\pi)$ Then $g \circ f(x)=\left(g_{1} \circ f(x), s_{0}^{q}(1)\right)$, $h \circ f(x)=\left(h_{1} \circ f(x), s_{0}^{q}(1)\right)$, and $(g+h) \circ f(x)=\left(g_{1} \circ f(x)+h_{1} \circ f(x), s_{0}^{q}(1)\right)$. Hence $f^{*}[g+h]=f^{*}[g]+f^{*}[h]$.

Definition 5.8. Suppose $\mathbf{F} \stackrel{i}{\rightarrow} \mathbf{E} \stackrel{p}{\rightarrow} \mathbf{B}$ is a Kan fiber sequence, and $f: \mathbf{B} \rightarrow \mathbf{L}_{\pi}(G, n+1)$ is a semi-simplicial mapping. Then $f$ induces maps making $\mathbf{F}, \mathbf{E}$, and $\mathbf{B}$ complexes over $\overline{\mathbf{W}}(\pi)$, and $i, p$, and $f$ maps over $\overline{\mathbf{W}}(\pi)$. Let $q: \mathbf{P}^{f} \rightarrow \mathbf{B}$ be the pullback over $\overline{\mathbf{W}}(\pi)$ by $f$ of the fibration $p: \mathbf{P}_{\pi}(G, n+1) \rightarrow \mathbf{L}_{\pi}(G, n+1)$. Note that $q: \mathbf{P}^{f} \rightarrow \mathbf{B}$ is a minimal fibration because $p: \mathbf{P}_{\pi}(G, n+1) \rightarrow \mathbf{L}_{\pi}(G, n+1)$ is.

Consider the following diagram

$$
\begin{aligned}
& \mathbf{F} \stackrel{i}{\longrightarrow} \mathbf{E} \\
& u \downarrow \quad \downarrow v \\
& \mathbf{K}(G, n) \longrightarrow \mathbf{P}^{f} \\
& q \downarrow \\
& \mathbf{B} \stackrel{f}{\longrightarrow} \mathbf{L}_{\pi}(G, n+1) \text {. }
\end{aligned}
$$

We say that $u: \mathbf{F} \rightarrow \mathbf{K}(G, n)$ is geometrically realized by $f$ if there exists $v:(\mathbf{E}, \mathbf{F}) \rightarrow\left(\mathbf{P}^{f}, \mathbf{K}(G, n)\right)$ such that $q v=p$ and $v \mid \mathbf{F} \cong u: \mathbf{F} \rightarrow \mathbf{K}(G, n)$. 
Let $\Sigma f=\{[u] \in[\mathbf{F}, \mathbf{K}(G, n)] \mid u$ is realized by $f\}$. It is easily checked that if $f \cong f^{\prime}$ over $\overline{\mathbf{W}}(\pi)$ then $\Sigma f=\Sigma f^{\prime}$, so $\Sigma f=\Sigma[f]_{\overline{\mathbf{W}}(\pi)}$.

Since $\mathbf{F} \rightarrow \overline{\mathbf{W}}(\pi)$ is trivial, by (5.7) the following map is a homomorphism:

$$
i^{*}:\left[\mathbf{E}, \mathbf{L}_{\pi}(G, n)\right]_{\overline{\mathbf{W}}(\pi)} \rightarrow\left[\mathbf{F}, \mathbf{L}_{\pi}(G, n)\right]_{\overline{\mathbf{W}}(\pi)}=[\mathbf{F}, \mathbf{K}(G, n)] .
$$

Theorem 5.9. $\Sigma f$ is a left coset of the subgroup $i^{*}\left[\mathbf{E}, \mathbf{L}_{\pi}(G, n)\right]_{\overline{\mathbf{W}}(\pi)}$ in $[\mathbf{F}, \mathbf{K}(G, n)]$.

Proof. (i) Consider the diagram:

$$
\begin{aligned}
{\left[(\mathbf{E}, \mathbf{F}),\left(\mathbf{B}, b_{0}\right)\right]_{\overline{\mathbf{W}}(\pi)} \stackrel{q_{*}}{\stackrel{i^{*}}{\leftrightarrows}}\left[(\mathbf{E}, \mathbf{F}),\left(\mathbf{P}^{f}, \mathbf{K}(G, n)\right)\right]_{\overline{\mathbf{W}}(\pi)} } \\
\left.\stackrel{i^{\prime}}{\rightarrow}[\mathbf{F}),\left(\mathbf{P}^{f}, \mathbf{K}(G, n)\right)\right]_{\overline{\mathbf{W}}(\pi)}=[\mathbf{F}, \mathbf{K}(G, n)] .
\end{aligned}
$$

Observe that $\Sigma f=i^{*} q_{*}{ }^{-1}[p]_{\overline{\mathbf{W}}(\pi)}$.

(ii) We prove the following:

Lemma 5.10. Let $(\mathbf{X}, \mathbf{A})$ be a pair over $\overline{\mathbf{W}}(\pi)$ then there is a group action of $\left[(\mathbf{X}, \mathbf{A}),\left(\mathbf{L}_{\pi}(G, n), \mathbf{K}(G, n)\right)\right]_{\overline{\mathbf{W}}(\pi)}$ on the set $\left[(\mathbf{X}, \mathbf{A}),\left(\mathbf{P}^{f}, \mathbf{K}(G, n)\right)\right]_{\overline{\mathbf{W}}(\pi)}$ such that for $\alpha, \beta \in\left[(\mathbf{X}, \mathbf{A}),\left(\mathbf{P}^{f}, \mathbf{K}(G, n)\right)\right]_{\overline{\mathbf{W}}(\pi)}, q_{*} \alpha=q_{*} \beta$ if and only if there exists $\eta \in\left[(\mathbf{X}, \mathbf{A}),\left(\mathbf{L}_{\pi}(G, n), \mathbf{K}(G, n)\right)\right] \overline{\mathbf{W}}_{(\pi)}$ such that $\eta \beta=\alpha$. The action is natural with respect to maps $(\mathbf{X}, \mathbf{A}) \rightarrow\left(\mathbf{X}^{\prime}, \mathbf{A}^{\prime}\right)$.

Proof. Recall that $f(b)=\left(f_{1}(b), \hat{b}(b)\right)$. It follows that

$$
\mathbf{L}_{\pi}(G, n) \times \overline{\mathbf{W}(\pi)} \mathbf{P}^{f}=\left\{(\omega, \hat{b}(b)) \times(\mu, \hat{b}(b), b) \mid \delta \mu=f_{1}(b)\right\} .
$$

Define $\gamma: \mathbf{L}_{\pi}(G, n) \times \times_{\overline{\mathbf{w}}(\pi)} \mathbf{P}^{f} \rightarrow \mathbf{P}^{f}$ by $\gamma((\omega, \hat{b}(b)) \times(\mu, \hat{b}(b), b))=(\omega+$ $\mu, \hat{b}(b), b)$. Notice that $\delta(\omega+\mu)=\delta \mu=f_{1}(b)$ so $\gamma$ is well defined. Note also that $q \gamma(x, y)=q(y)$ for all $(x, y) \in \mathbf{L}_{\pi}(G, n) \times \overline{\mathbf{W}(\pi)} \mathbf{P}^{f}$.

If $\beta:(\mathbf{X}, \mathbf{A}) \rightarrow\left(\mathbf{P}^{f}, \mathbf{K}(G, n)\right)$ and $\eta:(\mathbf{X}, \mathbf{A}) \rightarrow\left(\mathbf{L}_{\pi}(G, n), \mathbf{K}(G, n)\right)$ are maps over $\overline{\mathbf{W}}(\pi)$. Define $\eta \beta:(\mathbf{X}, \mathbf{A}) \rightarrow\left(\mathbf{P}^{f}, \mathbf{K}(G, n)\right)$ by $(\eta \beta)(x)=$ $\gamma(\eta(x), \beta(x))$. Notice that $q(\eta \beta(x))=q(\beta(x))$ for all $x$. It is easy to check that this action is defined on homotopy classes.

Let $g:(\mathbf{X}, \mathbf{A}) \rightarrow\left(\mathbf{X}^{\prime}, \mathbf{A}^{\prime}\right)$ be a map over $\overline{\mathbf{W}}(\pi)$. Consider the maps

$$
\begin{gathered}
g^{*}:\left[\left(\mathbf{X}^{\prime}, \mathbf{A}^{\prime}\right),\left(\mathbf{P}^{f}, \mathbf{K}(G, n)\right)\right]_{\overline{\mathbf{W}}(\pi)} \rightarrow\left[(\mathbf{X}, \mathbf{A}),\left(\mathbf{P}^{f}, \mathbf{K}(G, n)\right)\right]_{\overline{\mathbf{W}}(\pi)} ; \\
g^{*}:\left[\left(\mathbf{X}^{\prime}, \mathbf{A}^{\prime}\right),\left(\mathbf{L}_{\pi}(G, n), \mathbf{K}(G, n)\right)\right]_{\overline{\mathbf{W}}(\pi)} \rightarrow\left[(\mathbf{X}, \mathbf{A}),\left(\mathbf{L}_{\pi}(G, n), \mathbf{K}(G, n)\right)\right]_{\overline{\mathbf{W}}(\pi)} .
\end{gathered}
$$

Notice that

$$
\begin{aligned}
g^{*}(\eta \beta)(x) & =\gamma(\eta(g(x)), \beta(g(x))) \\
& =\left(g^{*} \eta\right)\left(g^{*} \beta\right)(x) .
\end{aligned}
$$


Suppose $q_{*}[\alpha]=q_{*}[\beta]$. By choice of representatives we may assume $q \circ \alpha=$ $q \circ \beta$. Now we can write:

$$
\begin{aligned}
& \alpha(x)=\left(\mu_{1}(x), \hat{x}(x), b(x)\right) ; \\
& \beta(x)=\left(\mu_{2}(x), \hat{x}(x), b(x)\right),
\end{aligned}
$$

where $\delta \mu_{1}(x)=f_{1}(b(x))=\delta \mu_{2}(x)$. It follows that $\delta\left(\mu_{1}(x)-\mu_{2}(x)\right)=0$ for all $x$. Hence $\mu_{1}(x)-\mu_{2}(x) \in \mathbf{K}(G, n)$ for all $x$. Define $\eta:(\mathbf{X}, \mathbf{A}) \rightarrow$ $\left(\mathbf{L}_{\pi}(G, n), \mathbf{K}(G, n)\right)$ by $\eta(x)=\left(\mu_{1}(x)-\mu_{2}(x), \hat{x}(x)\right)$. Then

$$
\begin{aligned}
(\eta \beta)(x) & =\left(\left(\mu_{1}(x)-\mu_{2}(x)\right)+\mu_{2}(x), \hat{x}(x), b(x)\right) \\
& =\left(\mu_{1}(x), \hat{x}(x), b(x)\right) \\
& =\alpha(x) .
\end{aligned}
$$

Thus $\eta \beta=\alpha$ as required.

(iii) We apply (ii) with $(\mathbf{X}, \mathbf{A})=(\mathbf{F}, \mathbf{F})$, and again with $\left(\mathbf{X}^{\prime}, \mathbf{A}^{\prime}\right)=(\mathbf{E}, \mathbf{F})$. Let $\alpha \in q_{*}{ }^{-1}[p]_{\overline{\mathbf{W}}(\pi)}$. By (ii)

$$
q_{*}^{-1}[p]_{\overline{\mathbf{W}}(\pi)}=\left\{\eta \alpha \mid \eta \in\left[(\mathbf{E}, \mathbf{F}),\left(\mathbf{L}_{\pi}(G, n), \mathbf{K}(G, n)\right)\right]_{\overline{\mathbf{W}}(\pi)}\right\} .
$$

Note that $\left[(\mathbf{E}, \mathbf{F}),\left(\mathbf{L}_{\pi}(G, n), \mathbf{K}(G, n)\right)\right]_{\overline{\mathbf{W}}(\pi)}=\left[\mathbf{E}, \mathbf{L}_{\pi}(G, n)\right]_{\overline{\mathbf{W}}(\pi)}$ because $\mathbf{F} \rightarrow \overline{\mathbf{W}}(\pi)$ is trivial. Consider the maps:

$$
\begin{aligned}
& i^{*}\left[(\mathbf{E}, \mathbf{F}),\left(\mathbf{P}^{f}, \mathbf{K}(G, n)\right)\right]_{\overline{\mathbf{W}}(\pi)} \rightarrow\left[(\mathbf{F}, \mathbf{F}),\left(\mathbf{P}^{f}, \mathbf{K}(G, n)\right)\right]_{\overline{\mathbf{W}}(\pi)} ; \\
& i^{*}\left[(\mathbf{E}, \mathbf{F}),\left(\mathbf{L}_{\pi}(G, n), \mathbf{K}(G, n)\right)\right]_{\overline{\mathbf{W}}(\pi)} \rightarrow\left[(\mathbf{F}, \mathbf{F}),\left(\mathbf{L}_{\pi}(G, n), \mathbf{K}(G, n)\right)\right]_{\overline{\mathbf{W}}(\pi)} .
\end{aligned}
$$

Note that:

$$
\begin{aligned}
{\left[(\mathbf{F}, \mathbf{F}),\left(\mathbf{P}^{f}, \mathbf{K}(G, n)\right)\right]_{\overline{\mathbf{W}}(\pi)} } & =[\mathbf{F}, \mathbf{K}(G, n)] ; \\
{\left[(\mathbf{F}, \mathbf{F}),\left(\mathbf{L}_{\pi}(G, n), \mathbf{K}(G, n)\right)\right]_{\overline{\mathbf{W}}(\pi)} } & =[\mathbf{F}, \mathbf{K}(G, n)] .
\end{aligned}
$$

Under these bijections the action of $\left[(\mathbf{F}, \mathbf{F}),\left(\mathbf{L}_{\pi}(G, n), \mathbf{K}(G, n)\right)\right]_{\overline{\mathbf{W}}(\pi)}$ on $\left[(\mathbf{F}, \mathbf{F}),\left(\mathbf{P}^{f}, \mathbf{K}(G, n)\right)\right]_{\overline{\mathbf{W}}(\pi)}$ is just ordinary addition in $[\mathbf{F}, \mathbf{K}(G, n)]$. Furthermore the second $i^{*}$ is the homomorphism $i^{*}\left[\mathbf{E}, \mathbf{L}_{\pi}(G, n)\right]_{\overline{\mathbf{W}}(\pi)} \rightarrow$ $[\mathbf{F}, \mathbf{K}(G, n)]$. Hence we have

$$
\begin{aligned}
i^{*} q_{*}{ }^{-1}[p]_{\overline{\mathbf{W}}(\pi)} & =\left\{i^{*}(\eta \alpha) \mid \eta \in\left[\mathbf{E}, \mathbf{L}_{\pi}(G, n)\right]_{\overline{\mathbf{W}}(\pi)}\right\} \\
& =\left\{\left(i^{*} \eta\right)+\left(i^{*} \alpha\right) \mid \eta \in\left[\mathbf{E}, \mathbf{L}_{\pi}(G, n)\right]_{\overline{\mathbf{W}}(\pi)}\right\} \\
& =i^{*} \alpha+i^{*}\left[\mathbf{E}, \mathbf{L}_{\pi}(G, n)\right]_{\overline{\mathbf{W}}(\pi)} .
\end{aligned}
$$


So by (i) we have $\Sigma f=i^{*} \alpha+i^{*}\left[\mathbf{E}, \mathbf{L}_{\pi}(G, n)\right]_{\overline{\mathbf{W}}(\pi)}$ as was to be shown.

Suppose that $\mathbf{F} \stackrel{i}{\rightarrow} \mathbf{E} \stackrel{p}{\rightarrow} \mathbf{B}$ is a Kan fiber sequence of one pointed complexes, with $\pi=\pi_{1}(\mathbf{B})$, and $\pi_{k}(\mathbf{F})=0$, for $k<n$. We make the identifications:

$$
\begin{aligned}
H^{n}(\mathbf{F} ; G) & \equiv[\mathbf{F}, \mathbf{K}(G, n)] \\
H^{n}\left(\mathbf{E} ; G_{\phi p_{*}}\right) & \equiv\left[\mathbf{E}, \mathbf{L}_{\pi}(G, n)\right]_{\overline{\mathbf{W}}(\pi)}
\end{aligned}
$$

and

$$
H^{n+1}\left(\mathbf{B} ; G_{\phi}\right) \equiv\left[\mathbf{B} ; \mathbf{L}_{\pi}(G, n+1)\right]_{\overline{\mathbf{W}}(\pi)} .
$$

If $\tau:\left[H^{n}(\mathbf{F} ; G)\right]^{\tilde{\phi}} \rightarrow H^{n+1}\left(\mathbf{B} ; G_{\phi}\right)$ is the transgression, then $\tau$ is a homomorphism with $\operatorname{ker}(\tau)=i^{*} H^{n}\left(\mathbf{E} ; G_{\phi p_{*}}\right)$. Thus if $\sigma$ is the suspension, and $\mu \in \operatorname{im}(\tau)$, then $\sigma(\mu)$ is a coset of $i^{*} H^{n}\left(\mathbf{E} ; G_{\phi p^{*}}\right)$ in $H^{n}(\mathbf{F} ; G)$.

Theorem 5.11. For $f$ as in (5.8) we have $\Sigma f=\sigma f$.

Proof. It suffices to find a common element in these two cosets. Let $[u] \in \Sigma f$ and consider the following commutative diagram

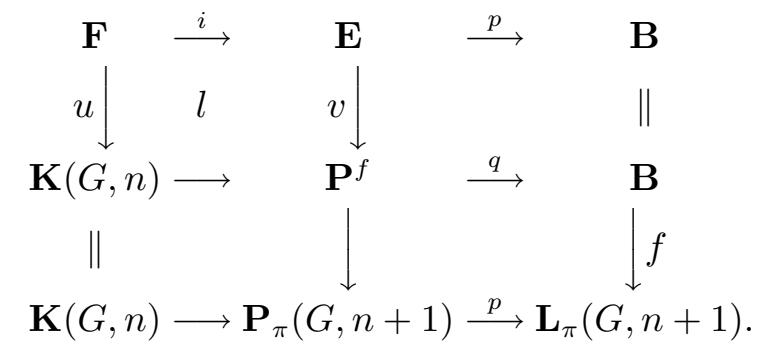

Let $\sigma, \sigma^{\prime \prime}$ be the suspensions corresponding to the first and third rows, respectively. Recall that $\iota_{n} \in \sigma^{\prime \prime}\left(\lambda_{n+1}\right)$. then, by the naturality of the suspension, we have $u^{*}\left(\iota_{n}\right) \in u^{*} \sigma^{\prime \prime}\left(\lambda_{n+1}\right) \subset \sigma f^{*}\left(\lambda_{n+1}\right)$ as required.

To construct the Moore-Postnikov tower for a connected Kan complex by the present method it remains only to show that the fundamental class of the fiber can always be realized.

Lemma 5.12. Suppose $n \geq 2$, and $\pi_{k}(\mathbf{F})=0$ for $k<n$. Let $f \in$ $H^{n}\left(\mathbf{F} ; \pi_{n}(\mathbf{F})\right)$ be the fundamental class, and $\phi: \pi_{1}(\mathbf{B}) \rightarrow \operatorname{Aut}\left(\pi_{n}(\mathbf{F})\right)$ be the action determined by the fibration. Then $f \in\left[H^{n}\left(\mathbf{F} ; \pi_{n}(\mathbf{F})\right)\right]^{\widetilde{\phi}}$. Hence $f$ is transgressive and can be realized.

Proof. See McClendon $[\mathbf{9}, 4.1]$. 
Now suppose $\mathbf{K}(G, n) \stackrel{i}{\rightarrow} \mathbf{E} \stackrel{p}{\rightarrow} \mathbf{B}$ is a Kan fiber sequence of one pointed Kan complexes. By (5.12) the fundamental class $\iota_{n} \in H^{n}(\mathbf{K}(G, n) ; G)$ is transgressive, and $\tau\left(\iota_{n}\right) \in H^{n+1}\left(\mathbf{B} ; G_{\phi}\right)$ corresponds to some class $[f] \in$ $\left[\mathbf{B} ; \mathbf{L}_{\pi}(G, n+1)\right]_{\overline{\mathbf{W}}(\pi)}$. Thus $\iota_{n} \in \sigma f=\Sigma f$. But $\iota_{n} \in H^{n}(\mathbf{K}(G, n) ; G)$ corresponds to the identity map $\mathbf{K}(G, n) \rightarrow \mathbf{K}(G, n)$, hence there exists a diagram

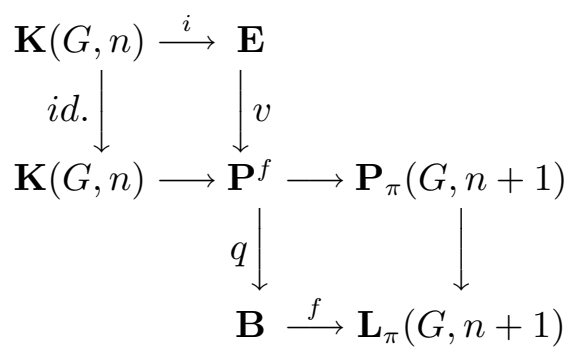

where $v:(\mathbf{E}, \mathbf{K}(G, n)) \rightarrow\left(\mathbf{P}^{f}, \mathbf{K}(G, n)\right), v \mid \mathbf{K}(G, n) \cong i d$, and $q v=p$. Comparing the long exact homotopy sequences of the two fibrations, we see that $v$ is a strong homotopy equivalence of fibrations.

Conversely it is easy to see that strongly equivalent $\mathbf{K}(G, n)$ fibrations over $\mathbf{B}$ determine homotopic maps $\mathbf{B} \rightarrow \mathbf{L}_{\pi}(G, n+1)$. Thus we have the following:

Theorem 5.13. Let $\phi: \pi \rightarrow \operatorname{Aut}(G)$, B be a one pointed Kan complex with $\pi_{1}(\mathbf{B})=\pi$. Then the set of equivalence classes of $\mathbf{K}(G, n)$ fibrations inducing $\phi$ are in one to one correspondence with $\left[\mathbf{B} ; \mathbf{L}_{\pi}(\mathbf{G} ; n+1)\right]_{\overline{\mathbf{W}}(\pi)} \cong$ $H^{n+1}\left(\mathbf{B} ; G_{\phi}\right)$.

See $[\mathbf{1 3}]$.

Definition 5.14. Let $\mathbf{K}$ be a connected minimal complex, $\pi_{n}=\pi_{n}(\mathbf{K})$, and let $\mathbf{X}=\left(X^{0}, X^{1}, \ldots, X^{n}, \ldots\right)$ be the natural Postnikov system of $\mathbf{K}$, see $[8,8.5]$. Then $X^{n-1}=\left(\mathbf{K}^{(n)}, p, \mathbf{K}^{(n-1)}\right)$ is a minimal fibration. Hence the fiber, $\mathbf{F}^{(n)}$ of $X^{n}$ is a minimal complex of homotopy type $\left(\pi_{n}, n\right)$. It follows that $\mathbf{F}^{(n)}$ is isomorphic to $\mathbf{K}\left(\pi_{n}, n\right)$. Thus we have a Kan fiber sequence $\mathbf{K}\left(\pi_{n}, n\right) \stackrel{i}{\rightarrow} \mathbf{K}^{(n)} \stackrel{p}{\rightarrow} \mathbf{K}^{(n-1)}$ of one pointed Kan complexes.

Let $\pi=\pi_{1}(\mathbf{K})=\pi_{1}\left(\mathbf{K}^{(n-1)}\right), \pi_{n}=\pi_{n}(\mathbf{K})=\pi_{n}\left(\mathbf{K}^{(n)}\right)$, and let $\phi$ : $\pi \rightarrow \operatorname{Aut}\left(\pi_{n}\right)$ be the action determined by $X^{n}$. The $n^{\text {th }} k$-invariant of $\mathbf{K}$ is defined by $k^{n}=t\left(\iota_{n}\right) \in H^{n+1}\left(\mathbf{K}^{(n-1)} ; \pi_{n \phi}\right)$. $k^{n}$ corresponds to a class $\left[f^{n}\right] \in\left[\mathbf{K}^{(n-1)}, \mathbf{L}_{\pi}\left(\pi_{n}, n+1\right)\right]_{\overline{\mathbf{W}}(\pi)} \cdot\left(\mathbf{P}^{f^{n}}, q, \mathbf{K}^{(n-1)}\right)$ is strongly equivalent to $\left(\mathbf{K}^{(n)}, p, \mathbf{K}^{(n-1)}\right)$, but these are both minimal fiber spaces, and a strong equivalence of minimal fiber spaces is an isomorphism, see [8, 12.8]. Thus the natural Postnikov system of $\mathbf{K}$ is determined by the homotopy groups of $\mathbf{K}$, the action of $\pi_{1}(\mathbf{K})$ on $\pi_{n}(\mathbf{K})$, for all $n$, and the $k$-invariants of $\mathbf{K}$. Since 
$\mathbf{K}$ is homotopy equivalent to the projective limit of it's natural Postnikov system it follows that the homotopy type of $\mathbf{K}$ is determined by it's homotopy groups the actions of $\pi_{1}(\mathbf{K})$, and it's $k$-invariants. Finally if $\mathbf{L}$ is an arbitrary connected Kan complex, $\mathbf{L}$ contains a minimal subcomplex $\mathbf{K}$ which is a strong deformation retract, see [8, 9.9]. Thus the homotopy type of $\mathbf{L}$ is determined by the homotopy type of $\mathbf{K}$.

Example 5.15. If we consider $\overline{\mathbf{W}}(\pi)$ as a complex over itself via the identity map, then the cannonical section $s_{0}: \overline{\mathbf{W}}(\pi) \rightarrow \mathbf{L}_{\pi}(G, n+1)$ is a map over $\overline{\mathbf{W}}(\pi)$ and

$$
\begin{aligned}
\mathbf{P}^{s_{0}} & =\left\{(\mu, x, x) \in \mathbf{P}_{\pi}(G, n+1) \times \overline{\mathbf{W}}(\pi) \overline{\mathbf{W}}(\pi) \mid \delta \mu=0\right\} \\
& =\mathbf{L}_{\pi}(G, n) \times \overline{\mathbf{W}(\pi)} \overline{\mathbf{W}}(\pi) .
\end{aligned}
$$

Define $v: \mathbf{L}_{\pi}(G, n) \rightarrow \mathbf{P}^{s_{0}}$ by $v(\mu, x)=(\mu, x, x)$ and notice that $q(v(\mu, x))=$ $p_{2}(\mu, x)$. We have the following commutative diagram

$$
\begin{array}{ccc}
\mathbf{K}(G, n) \stackrel{i}{\rightarrow} \mathbf{L}_{\pi}(G, n) & \\
i d . \| & \downarrow v & \\
\mathbf{K}(G, n) \stackrel{i}{\rightarrow} & \mathbf{P}^{s_{0}} & \rightarrow \mathbf{P}_{\pi}(G, n+1) \\
& \frac{q \downarrow}{\mathbf{W}}(\pi) & \stackrel{s_{0}}{\rightarrow} \mathbf{L}_{\pi}(G, n+1)
\end{array}
$$

$s_{0}$ corresponds to $0 \in H^{n+1}\left(\overline{\mathbf{W}}(\pi) ; G_{\phi}\right)$, therefore $\mathbf{L}_{\pi}(G, n)$ has zero $k$ invariant.

\section{Obstruction Theory.}

Let $(\mathbf{X}, \mathbf{A})$ be a one pointed semi-simplicial pair, $\mathbf{Y}$ be a connected Kan complex. We develop a twisted coefficient obstruction theory for the extension of maps $f: \mathbf{A} \rightarrow \mathbf{Y}$ and homotopies $f_{0}\left|\mathbf{A} \cong f_{1}\right| \mathbf{A}$, (rel $\left.x_{0}\right)$ where $f_{0}, f_{1}: \mathbf{X} \rightarrow \mathbf{Y}$.

This section generalizes the obstruction theory of P. Olum [10], where $\mathbf{X}$ and $\mathbf{Y}$ are total singular complexes of arc connected topological spaces.

Lemma 6.1. W.L.O.G. it suffices to consider codomains $\mathbf{Y}$ such that $\mathbf{Y}$ is the minimal subcomplex of the total singular complex $\mathbf{S}(\mathbf{W})$ of an arc connected topological space $\mathbf{W}$.

Proof. Let $\mathbf{Y}$ be a connected Kan complex and let $\mathbf{M} \leq \mathbf{Y}$ be the minimal subcomplex of $\mathbf{Y}$, see $[8,9.9]$. Let $\mathbf{M} \stackrel{i}{\rightarrow} \mathbf{Y} \stackrel{r}{\rightarrow} \mathbf{M}$ be the diagram defining $\mathbf{M}$ as a strong deformation retract of $\mathbf{Y}$. Then $r \circ i=1_{M}$, and there exists 
$H: \mathbf{Y} \times \mathbf{I} \rightarrow \mathbf{Y}$ such that $H \circ i_{0}=i \circ r, H \circ i_{1}=1_{Y}$, and $H(m, \sigma)=m$, for all $(m, \sigma) \in \mathbf{M} \times \mathbf{I}$.

If $f_{0}: \mathbf{A} \rightarrow \mathbf{Y}$, let $\phi_{0}=r \circ f_{0}: \mathbf{A} \rightarrow \mathbf{M}$. Suppose $\phi_{0}$ has an extension $\phi_{n}: \mathbf{X}^{(n)} \cup \mathbf{A} \rightarrow \mathbf{M}$. Then $i \circ \phi_{n}: \mathbf{X}^{(n)} \cup \mathbf{A} \rightarrow \mathbf{Y}$ with $i \circ \phi_{n} \mid \mathbf{A}=i \circ \phi_{0}=i \circ r \circ f_{0}$. Define $K: \mathbf{A} \times \mathbf{I} \rightarrow \mathbf{Y}$ by $K(a, \sigma)=H\left(f_{0}(a), \sigma\right)$. Then $K\left(a, s_{0}^{q}(0)\right)=i \circ \phi_{0}(a)$ and $K\left(a, s_{0}^{q}(1)\right)=f_{0}(a)$. Now define $F_{n}:\left(\mathbf{X}^{(n)} \times(0)\right) \cup(\mathbf{A} \times \mathbf{I}) \rightarrow \mathbf{Y}$ by

$$
F_{n}(x, \sigma)= \begin{cases}K(x, \sigma) & \text { if }(x, \sigma) \in \mathbf{A} \times \mathbf{I} \\ i \circ \phi_{n}(x) & \text { if } \quad(x, \sigma) \in \mathbf{X} \times(0) .\end{cases}
$$

Then $F_{n}$ extends over $\left(\mathbf{X}^{(n)} \times \mathbf{I}\right) \cup(\mathbf{A} \times \mathbf{I})$ by the homotopy extension property. Define $f_{n}: \mathbf{X}^{(n)} \cup \mathbf{A} \rightarrow \mathbf{Y}$ by $f_{n}(x)=F_{n}\left(x, s_{0}^{q}(1)\right)$. Then for $a \in \mathbf{A}$ we have $f_{n}(a)=f_{0}(a)$, hence $f_{n}$ is an extension of $f_{0}$.

Conversely suppose $f_{0}$ has an extension $f_{n}: \mathbf{X}^{(n)} \cup \mathbf{A} \rightarrow \mathbf{Y}$. Define $\phi_{n}=r \circ f_{n}: \mathbf{X}^{(n)} \cup \mathbf{A} \rightarrow \mathbf{M}$ Then $\phi_{n}$ is an extension of $\phi_{0}$.

Therefore $f_{0}$ extends to $\mathbf{X}^{(n)} \cup \mathbf{A}$ if and only if $\phi_{0}$ does. Hence it suffices to consider codomains $\mathbf{Y}$ such that $\mathbf{Y}$ is a connected minimal complex.

Now let $\mathbf{Y}$ be a connected minimal complex. We have a homotopy equivalence $\Psi(\mathbf{Y}): \mathbf{Y} \rightarrow \mathbf{S}(\mathbf{T}(\mathbf{Y}))$, see $[8,16.6]$. Let $\mathbf{M} \leq \mathbf{S}(\mathbf{T}(\mathbf{Y}))$ be the minimal subcomplex. Then $r \circ \Psi(\mathbf{Y}): \mathbf{Y} \rightarrow \mathbf{M}$ is a homotopy equivalence of minimal complexes, but such a map is an isomorphism, see [8, 9.7]. This proves the Lemma.

If $\mathbf{Y} \leq \mathbf{S}(\mathbf{W})$ is the minimal subcomplex of the total sigular complex of an arc connected topological space $\mathbf{W}$, then $\mathbf{Y}$ is a one pointed complex. Let $y_{0}: \Delta_{0} \rightarrow \mathbf{W}$ be the unique 0 -simplex of $\mathbf{Y}$, and let $w_{0}=y\left(\Delta_{0}\right) \in \mathbf{W}$. If $y \in Y_{q}$ is an arbitrary $q$-simplex then $y: \Delta_{q} \rightarrow \mathbf{W}$, and if $v_{i}$ is the $i^{t h}$ vertex of $\Delta_{q}$ then $y\left(v_{i}\right)=w_{0}, \quad 0 \leq i \leq q$.

Definition 6.2. Let $\Delta[n]$ be the standard semi-simplicial n-simplex, with generator $\sigma_{n}$, and let $\sigma^{(i)}=\partial_{i} \sigma_{n}, 0 \leq i \leq n$. Let $\partial \Delta[n]$ be the subcomplex of $\Delta[n]$ generated by the $\sigma^{(i)}$. Suppose $f: \partial \Delta[n] \rightarrow \mathbf{Y}$ is a semisimplicial map. If $i: \mathbf{Y} \rightarrow \mathbf{S}(\mathbf{W})$ is the inclusion then $i \circ f: \partial \Delta[n] \rightarrow \mathbf{S}(\mathbf{W})$. Let $\phi(i \circ f): \partial \Delta_{n} \rightarrow \mathbf{W}$ be the adjoint map, see [8, 16.1]. If $e_{i}: \Delta_{n-1} \rightarrow \Delta_{n}$ is the $i^{\text {th }}$ face, then $\phi(i \circ f) \circ e_{i}=f\left(\sigma^{(i)}\right): \Delta_{n-1} \rightarrow \mathbf{W}$. Now $f\left(\sigma^{(i)}\right) \in Y_{n-1}$, for all $i$, so if $v_{0}$ is the leading vertex of $\Delta_{n}$ then $\phi(i \circ f):\left(\partial \Delta_{n}, v_{0}\right) \rightarrow\left(\mathbf{W}, w_{0}\right)$. Therefore $\phi(i \circ f)$ determines an element $\alpha(f) \in \pi_{n-1}\left(\mathbf{W}, w_{0}\right)$.

Lemma 6.3. $\quad f$ extends over $\Delta[n]$ if and only if $\alpha(f)$ is trivial in $\pi_{n-1}\left(\mathbf{W}, w_{0}\right)$.

Proof. If $\alpha(f)$ is trivial, then $\phi(i \circ f)$ extends to a continuous function $\tilde{\phi}$ : $\Delta_{n} \rightarrow \mathbf{W}$. Note $\tilde{\phi} \in S_{n}(\mathbf{W})$. If $r: \mathbf{S}(\mathbf{W}) \rightarrow \mathbf{Y}$ is the retraction, define a 
$\operatorname{map} \tilde{f}: \Delta[n] \rightarrow \mathbf{Y}$ by $\tilde{f}\left(\sigma_{n}\right)=r(\tilde{\phi}) \in \mathbf{Y}$. Then

$$
\begin{aligned}
\partial_{i} \tilde{f}\left(\sigma_{n}\right) & =r\left(\partial_{i} \tilde{\phi}(i \circ f)\right) \\
& =r\left(\phi(i \circ f) \circ e_{i}\right) \\
& =f\left(\partial_{i} \sigma_{n}\right) .
\end{aligned}
$$

Thus $\tilde{f}$ is an extension of $f$.

Conversely, if $f$ extends to $\tilde{f}: \Delta[n] \rightarrow \mathbf{Y}$ then $\phi(i \circ f)=\phi(i \circ \tilde{f}) \mid \partial \Delta_{n}$ hence $\alpha(f)$ is trivial.

Theorem 6.4. Let $f_{0}: \mathbf{A} \rightarrow \mathbf{Y}$ be a semi-simplicial map and suppose $\theta: \pi_{1}(\mathbf{X}) \rightarrow \pi_{1}(\mathbf{Y})$ is a homomorphism. Then $f_{0}$ has an extension $f_{2}: \mathbf{X}^{(2)} \cup$ $\mathbf{A} \rightarrow \mathbf{Y}$ inducing $\theta$ on fundamental groups if and only if $f_{0}(a)$ represents $\theta[a]$ whenever $a \in A_{1}$.

Proof. If an extension $f_{2}$ exists inducing $\theta$ on fundamental groups, then $f_{0}(a)=f_{2}(a)$ represents $\theta[a]$ whenever $a \in A_{1}$.

Conversely suppose $f_{0}(a)$ represents $\theta[a]$ whenever $a \in A_{1}$. Extend $f_{0}$ to $f_{1}: \mathbf{X}^{(1)} \cup \mathbf{A} \rightarrow \mathbf{Y}$ as follows. If $x \in X_{1}-A_{1}$ is nondegenerate, let $y \in Y_{1}$ represent $\theta[x]$ and define $f_{1}(x)=y$.

Now we show that $f_{1}$ can be extended to $f_{2}: \mathbf{X}^{(2)} \cup \mathbf{A} \rightarrow \mathbf{Y}$. Let $x \in$ $X_{2}-A_{2}$ be nondegenerate. Let $y_{i}=f_{1}\left(\partial_{i} x\right), 0 \leq i \leq 2$. Let $\bar{x}: \Delta[2] \rightarrow \mathbf{X}^{(2)}$ be the map determined by $\bar{x}\left(\sigma_{2}\right)=x$, and let $\partial \bar{x}: \partial \Delta[2] \rightarrow \mathbf{X}^{(1)}$ be $\bar{x}$ restricted to $\partial \Delta[2]$. Then the composite map $\partial \Delta[2] \stackrel{\partial \bar{x}}{\rightarrow} \mathbf{X}^{(1)} \stackrel{f_{1}}{\rightarrow} \mathbf{Y}$ determines an element $\alpha\left(f_{1}, x\right) \in \pi_{1}\left(\mathbf{W}, w_{0}\right)$ as in (6.2). But

$$
\begin{aligned}
\alpha\left(f_{1}, x\right) & =\left[y_{0}\right]\left[y_{2}\right]\left[y_{1}\right]^{-1} \\
& =\theta\left(\left[\partial_{0} x\right]\left[\partial_{2} x\right]\left[\partial_{1} x\right]^{-1}\right) \\
& =1
\end{aligned}
$$

because $\left[\partial_{0} x\right]\left[\partial_{2} x\right]=\left[\partial_{1} x\right]$ by the definition of multiplication in $\pi_{1}(\mathbf{X})$, see $[\mathbf{8}, 4.1]$. Thus $f_{1} \circ \partial \bar{x}$ extends over $\Delta[2]$ by $(6.3)$, so $f_{1}$ extends over $x$. It is clear from the construction that $f_{2}$ will induce $\theta$ on fundamental groups.

Definition 6.5. Suppose $f_{0}$ has an extension $f_{n-1}: \mathbf{X}^{(n-1)} \cup \mathbf{A} \rightarrow \mathbf{Y}$, $n \geq 3$, inducing $\theta$ on fundamental groups. Let $x \in X_{n}$. Then the composite map $\partial \Delta[n] \stackrel{\partial \bar{x}}{\rightarrow} \mathbf{X}^{(n-1)} \stackrel{f_{n-1}}{\rightarrow} \mathbf{Y}$ determines an element $\alpha\left(f_{n-1}, x\right) \in$ $\pi_{n-1}\left(\mathbf{W}, w_{0}\right)$ as in (6.3), such that $f_{n-1}$ extends over $x$ if and only if $\alpha\left(f_{n-1}, x\right)=0$. Notice that $\alpha\left(f_{n-1}, x\right)=0$ whenever $\quad x \in \mathbf{X}^{(n-1)} \cup \mathbf{A}$. 
Thus we obtain a normalized cochain $z\left(f_{n-1}\right) \in C^{n}\left(\mathbf{X}, \mathbf{A} ; \pi_{n-1}(\mathbf{W})_{\phi \theta}\right)$ defined by $z\left(f_{n-1}\right)(x)=\alpha\left(f_{n-1}, x\right)$. Where $\phi: \pi_{1}(\mathbf{Y}) \rightarrow \operatorname{Aut}\left(\pi_{n-1}\left(\mathbf{W}, w_{0}\right)\right)$ is the action determined by the action of $\pi_{1}(\mathbf{W})$ on $\pi_{n-1}(\mathbf{W})$.

We require the following to show that $z\left(f_{n-1}\right)$ is a cocycle.

Lemma 6.6. Let $\partial \Delta_{r+1}$ be the boundary of the geometric $(r+1)$ simplex, let $j$ be a fixed integer $0 \leq j \leq r+1$, let $\sigma_{j}$ be the $j^{\text {th }}$-face of $\Delta_{r+1}$, let $p_{0} \in \partial \Delta_{r+1}$ be the leading vertex of $\Delta_{r+1}$, and let $p_{1}$ be the leading vertex of $\sigma_{j}$. Suppose that $f, f^{\prime}: \partial \Delta_{r+1} \rightarrow \mathbf{W}$ such that $f\left(p_{0}\right)=f\left(p_{1}\right)=f^{\prime}\left(p_{0}\right)=f^{\prime}\left(p_{1}\right)=w_{0}$, $f^{\prime}\left|\partial \Delta_{r+1}-\sigma_{j}=f\right| \partial \Delta_{r+1}-\sigma_{j}$ and $f^{\prime} \mid \sigma_{j}$ is an alteration of $f \mid \sigma_{j}$ by the homotopy element $\beta \in \pi_{r}\left(\mathbf{W}, w_{0}\right)$. If $f$ and $f^{\prime}$ determine $\alpha_{0}$ and $\alpha_{0}^{\prime}$ in $\pi_{r}\left(\mathbf{W}, w_{0}\right)$ with $p_{0}$ as basepoint then $\alpha_{0}^{\prime}=\alpha_{0}+(-1)^{j} \omega_{01} \beta$ where $\omega_{01}$ is the class in $\pi_{r}\left(\mathbf{W}, w_{0}\right)$ containing the image under $f$ of all paths in $\partial \Delta_{r+1}$ from $p_{0}$ to $p_{1}$.

Proof. See $[\mathbf{1 0}, 6.5]$. Note that $\omega_{01}$ is trivial unless $j=0$, so we have

$$
\alpha_{0}^{\prime}= \begin{cases}\alpha_{0}+\omega_{01} \beta & \text { if } j=0 \\ \alpha_{0}+(-1)^{j} \beta & \text { if } j>0\end{cases}
$$

Theorem 6.7. $z\left(f_{n-1}\right) \in C^{n}\left(\mathbf{X}, \mathbf{A} ; \pi_{n-1}\left(\mathbf{W}, w_{0}\right)_{\phi \theta}\right)$ is a cocycle.

Proof. Let $e_{i}: \Delta_{q-1} \rightarrow \Delta_{q}$, imbed $\Delta_{q-1}$ as the $i^{\text {th }}$ face of $\Delta_{q}, q \geq 1$. Suppose $x \in X_{n+1}$, let $\bar{x}: \Delta[n+1] \rightarrow \mathbf{X}^{(n+1)}$ be the map determined by $x$, and let $\bar{x}^{(n-1)}$ be $\bar{x}$ restricted to the (n-1) skeleton of $\Delta[n+1]$. Then we have a composite map $i \circ f_{n-1} \circ \bar{x}^{(n-1)}: \Delta[n+1]^{(n-1)} \rightarrow \mathbf{S}(\mathbf{W})$. Let $\Sigma^{(n-1)}$ be the (n-1)-skeleton of $\Delta_{n+1}$. Define $F: \Sigma^{(n-1)} \rightarrow \mathbf{W}$ to be the adjoint map $F=\phi\left(i \circ f_{n-1} \circ \bar{x}^{(n-1)}\right)$. Notice that $F \circ e_{j} \circ e_{i}=f_{n-1}\left(\partial_{i} \partial_{j} x\right): \Delta_{n-1} \rightarrow \mathbf{W}$.

Next define $F^{\prime}: \Sigma^{(n-1)} \rightarrow \mathbf{W}$ by setting $F^{\prime} \mid e_{n+1} e_{i}\left(\Delta_{n-1}\right)$ equal to an alteration of $F \mid e_{n+1} e_{i}\left(\Delta_{n-1}\right)$ by the homotopy element $(-1)^{n+1} z\left(f_{n-1}\right)\left(\partial_{i} x\right) \in$ $\pi_{n-1}\left(\mathbf{W}, w_{0}\right), \quad 0 \leq i \leq n+1$ and setting $F^{\prime}=F$ elsewhere.

Let $\alpha_{i}\left(F^{\prime}\right) \in \pi_{n-1}\left(\mathbf{W}, w_{0}\right)$ denote the homotopy element determined by $F^{\prime} \mid \partial e_{i}\left(\Delta_{n}\right), 0 \leq i \leq n+1$. Then for $0 \leq i \leq n$, we have

$$
\begin{aligned}
\alpha_{i}\left(F^{\prime}\right) & =z\left(f_{n-1}\right)\left(\partial_{i} x\right)+(-1)^{n}(-1)^{n+1} z\left(f_{n-1}\right)\left(\partial_{i} x\right) \\
& =0
\end{aligned}
$$

by (6.6), since $F \mid \partial e_{i}\left(\Delta_{n}\right)$ induces the element $z\left(f_{n-1}\right)\left(\partial_{i} x\right)$, and 
$e_{n+1} e_{i}\left(\Delta_{n-1}\right)=e_{i} e_{n}\left(\Delta_{n-1}\right)$ is the $n^{\text {th }}$ face of $e_{i}\left(\Delta_{n}\right)$. Furthermore

$$
\begin{aligned}
\alpha_{n+1}\left(F^{\prime}\right)= & z\left(f_{n-1}\right)\left(\partial_{n+1} x\right)+(-1)^{n+1} \omega_{01} z\left(f_{n-1}\right)\left(\partial_{0} x\right) \\
& +\sum_{i=1}^{n}(-1)^{i}(-1)^{n+1} z\left(f_{n-1}\right)\left(\partial_{i} x\right) \\
= & (-1)^{n+1}\left[\left(\delta z\left(f_{n-1}\right)\right)(x)\right]
\end{aligned}
$$

by (6.6), since $F \mid \partial e_{n+1}\left(\Delta_{n}\right)$ induces the homtopy element $z\left(f_{n-1}\right)\left(\partial_{n+1} x\right)$, $e_{n+1} e_{i}\left(\Delta_{n-1}\right)$ is the $i^{t h}$ face of $e_{n+1}\left(\Delta_{n}\right)$, and $F^{\prime} \mid e_{n+1} e_{i}\left(\Delta_{n-1}\right)$ is an alteration of $F \mid e_{n+1} e_{i}\left(\Delta_{n-1}\right)$ by $(-1)^{n+1} z\left(f_{n-1}\right)\left(\partial_{i} x\right)$. We also use the fact that $\omega_{01}$ is represented by $f_{n-1}\left(\partial_{2} \ldots \partial_{n+1} x\right)$, whence $\omega_{01}=\theta\left[\partial_{2} \ldots \partial_{n+1} x\right] \in \pi_{1}(\mathbf{W})$.

$\alpha_{i}\left(F^{\prime}\right)=0,0 \leq i \leq n$, implies that $F^{\prime}$ extends over $e_{i}\left(\Delta_{n}\right), 0 \leq i \leq n$. $e_{0}\left(\Delta_{n}\right) \cup e_{1}\left(\Delta_{n}\right) \cup \ldots \cup e_{n}\left(\Delta_{n}\right)$ forms a disc in $\partial \Delta_{n+1}$ with boundary (except for orientation) equal to $\partial e_{n+1}\left(\Delta_{n}\right)$. Therefore $F^{\prime} \mid \partial e_{n+1}\left(\Delta_{n}\right)$ extends over a disc in $\partial \Delta_{n+1}$, so $\alpha_{n+1}\left(F^{\prime}\right)=0$, hence $\left(\delta z\left(f_{n-1}\right)\right)(x)=0$. Since this holds for all $x \in X_{n+1}, z\left(f_{n-1}\right)$ is a cocycle in $C^{n}\left(\mathbf{X}, \mathbf{A} ; \pi_{n-1}\left(\mathbf{W}, w_{0}\right)_{\phi \theta}\right)$.

Definition 6.8. The cocycle $z\left(f_{n-1}\right)$ determines a cohomology class $h\left(f_{n-1}\right) \in H^{n}\left(\mathbf{X} ; \mathbf{A} ; \pi_{n-1}\left(\mathbf{W}, w_{0}\right)_{\phi \theta}\right)$ called the obstruction class of $f_{n-1}$.

Lemma 6.9. $z\left(f_{n-1}\right)$ is natural with respect to mappings of one pointed pairs.

Proof. Let $g:(\mathbf{U}, \mathbf{B}) \rightarrow(\mathbf{X}, \mathbf{A})$ be a map of one pointed pairs. Then $f_{n-1} \circ g: \mathbf{U}^{(n-1)} \cup \mathbf{B} \rightarrow \mathbf{Y}$ determines the obstruction cocycle $z\left(f_{n-1} \circ g\right)$ where $z\left(f_{n-1} \circ g\right)(u)$ is the homotopy element induced by the map $\phi(i \circ$ $\left.f_{n-1} \circ g \circ \partial \bar{u}\right) \cdot \overline{g(u)}=g \circ \bar{u}$ so $g \circ \partial \bar{u}=\partial \overline{g(u)}$, thus

$$
\begin{aligned}
z\left(f_{n-1} \circ g\right)(u) & =\left[\phi\left(i \circ f_{n-1} \partial \overline{g(u)}\right)\right] \\
& =z\left(f_{n-1}\right)(g(u)) \\
& =\left(g^{*} z\left(f_{n-1}\right)\right)(u) .
\end{aligned}
$$

Hence $z\left(f_{n-1} \circ g\right)=g^{*} z\left(f_{n-1}\right)$.

Lemma 6.10. Suppose $n \geq 2$, and $f_{n}, f_{n}^{\prime}: \mathbf{X}^{(n)} \cup \mathbf{A} \rightarrow \mathbf{Y}$ such that $f_{n}\left|\mathbf{X}^{(n-1)} \cup \mathbf{A} \cong f_{n}^{\prime}\right| \mathbf{X}^{(n-1)} \cup \mathbf{A} \quad\left(\right.$ rel $\left.x_{0}\right)$. Where we require that $f_{n}$ and $f_{n}^{\prime}$ both induce $\theta$ on fundamental groups if $n=2$. Then $h(f)=h\left(f^{\prime}\right) \in$ $H^{n+1}\left(\mathbf{X}, \mathbf{A} ; \pi_{n}\left(\mathbf{W}, w_{0}\right)_{\phi \theta}\right)$.

Proof. Let $K: \mathbf{X}^{(n-1)} \times \mathbf{I} \cup \mathbf{A} \times \mathbf{I} \rightarrow \mathbf{Y}$ be a homotopy $f_{n} \mid \mathbf{X}^{(n-1)} \cup \mathbf{A} \cong$ $f_{n}^{\prime} \mid \mathbf{X}^{(n-1)} \cup \mathbf{A}\left(\right.$ rel $\left.x_{0}\right)$. Define $H: \mathbf{X}^{(n)} \times(0) \cup\left(\mathbf{X}^{(n-1)} \times \mathbf{I} \cup \mathbf{A} \times \mathbf{I}\right) \cup \mathbf{X}^{(n)} \times(1) \rightarrow$ 
$\mathbf{Y}$ by

$$
H(x, \sigma)= \begin{cases}f(x) & \text { if }(x, \sigma) \in \mathbf{X}^{(n)} \times(0) ; \\ K(x, \sigma) & \text { if }(x, \sigma) \in \mathbf{X}^{(n-1)} \times \mathbf{I} \cup \mathbf{A} \times \mathbf{I} \\ f^{\prime}(x) & \text { if }(x, \sigma) \in \mathbf{X}^{(n)} \times(1) .\end{cases}
$$

It is straight forward to check that $\mathrm{H}$ extends over $(\mathbf{X} \times \mathbf{I})^{(n)} \cup \mathbf{A} \times \mathbf{I}$. Since $H\left(x_{0} \times \mathbf{I}\right)=\left\{y_{0}\right\}$ we may think of $\mathrm{H}$ as being defined on the one pointed complex $\left([\mathbf{X} \times \mathbf{I}]^{(n)} \cup \mathbf{A} \times \mathbf{I}\right) /\left(x_{0} \times \mathbf{I}\right)$. It follows that $\mathrm{H}$ induces an obstruction class $h(H) \in H^{n+1}\left(\mathbf{X} \times \mathbf{I}, \mathbf{A} \times \mathbf{I} ; \pi_{n}\left(\mathbf{W}, w_{0}\right)_{\phi \bar{\theta}}\right)$. Where $\bar{\theta}: \pi_{1}(\mathbf{X} \times \mathbf{I}) \rightarrow \pi_{1}(\mathbf{Y})$ is the homomorphism induced by $\mathrm{H}$. By (6.9) we have

$$
\begin{aligned}
h(f) & =i_{0}^{*} h(H) \\
& =i_{1}^{*} h(H) \\
& =h\left(f^{\prime}\right) .
\end{aligned}
$$

Corollary 6.11. The obstruction class $h\left(f_{n}\right)$ depends only on the based homotopy class of $f_{n}$.

Definition 6.12. Alteration of a semi-simplicial mapping. Let $x$ be an $n$-simplex of $\mathbf{X}$. Given a map $f: \mathbf{X}^{(n)} \cup \mathbf{A} \rightarrow \mathbf{Y}, n \geq 2$. We may consider alterations of the singular $n$-simplex $f(x): \Delta_{n} \rightarrow \mathbf{W}$ by homotopy elements $\alpha \in \pi_{n}\left(\mathbf{W}, w_{0}\right)$.

Note that if $y: \Delta_{n} \rightarrow \mathbf{W}$ is an $n$-simplex of $\mathbf{Y}$, and $u: \Delta_{n} \rightarrow \mathbf{W}$ is an alteration of $y$ then $\partial_{i} u=\partial_{i} y \in Y_{n-1}$, for all $i$, and since $\mathbf{Y}$ is the minimal subcomplex of $\mathbf{S}(\mathbf{W})$ it follows that there exists $y^{\prime} \in Y_{n}$ such that $y^{\prime}$ is homotopic to $u$ (rel $\partial \Delta_{n}$ ). Thus we may assume that an alteration of an $n$-simplex of $\mathbf{Y}$ is again an $n$-simplex of $\mathbf{Y}$.

Now, given an $n$-cochain $c^{n} \in C^{n}\left(\mathbf{X}, \mathbf{A} ; \pi_{n}\left(\mathbf{W}, w_{0}\right)_{\phi \theta}\right)$ we define a new semi-simplicial mapping $f_{a}: \mathbf{X}^{(n)} \cup \mathbf{A} \rightarrow \mathbf{Y}$ as follows. For $x \in X_{n}$ set

$$
f_{a}(x)= \begin{cases}f(x) & \text { if } c^{n}(x)=0 \\ f(x)^{\prime} & \text { if } c^{n}(x) \neq 0\end{cases}
$$

Where $f(x)^{\prime}$ is an alteration of $f(x)$ by the homotopy element $c^{n}(x)$.

We commpute the effect of an alteration on the obstruction cocycle.

Lemma 6.13. Given $f: \mathbf{X}^{(n)} \cup \mathbf{A} \rightarrow \mathbf{Y}, n \geq 2$, and an alteration $f_{a}$ of $f$ by $c^{n} \in C^{n}\left(\mathbf{X}, \mathbf{A} ; \pi_{n}\left(\mathbf{W}, w_{0}\right)_{\phi \theta}\right)$ then $z\left(f_{a}\right)-z(f)=\delta c^{n}$.

Proof. $z(f)(x)$, and $z\left(f_{a}\right)(x)$ are induced by $\phi(i \circ f \circ \partial \bar{x})$ and $\phi\left(i \circ f_{a} \circ \partial \bar{x}\right)$ respectively. Now $\phi(i \circ f \circ \partial \bar{x}) \circ e_{i}=f\left(\partial_{i} x\right)$ and $\phi\left(i \circ f_{a} \circ \partial \bar{x}\right) \circ e_{i}=$ 
$f_{a}\left(\partial_{i} x\right)$. But $f_{a}\left(\partial_{i} x\right)$ is an alteration of $f\left(\partial_{i} x\right)$ by $c^{n}\left(\partial_{i} x\right)$. Therefore by $(6.9) z\left(f_{a}\right)(x)=z(f)(x)+\omega_{01} c^{n}\left(\partial_{0} x\right)+\sum_{i=1}^{n+1}(-1)^{i} c^{n}\left(\partial_{i} x\right)$, whence $z\left(f_{a}\right)(x)-$ $z(f)(x)=\left(\delta c^{n}\right)(x)$.

Theorem 6.14. If $f: \mathbf{X}^{(n)} \cup \mathbf{A} \rightarrow \mathbf{Y}, n \geq 2$, induces $\theta: \pi_{1}(\mathbf{X}) \rightarrow \pi_{1}(\mathbf{Y})$, then there exists $g: \mathbf{X}^{(n+1)} \cup \mathbf{A} \rightarrow \mathbf{Y}$ inducing $\theta$, such that $g \mid \mathbf{X}^{(n-1)} \cup \mathbf{A}=$ $f \mid \mathbf{X}^{(n-1)} \cup \mathbf{A}$ if and only if $h(f)=0 \in H^{n+1}\left(\mathbf{X}, \mathbf{A} ; \pi_{n}\left(\mathbf{W}, w_{0}\right)_{\phi \theta}\right)$.

Proof. Suppose $h(f)=0$.

Then $z(f)=\delta c^{n}$ for some $c^{n} \in C^{n}\left(\mathbf{X}, \mathbf{A} ; \pi_{n}\left(\mathbf{W}, w_{0}\right)_{\phi \theta}\right)$. Let $f_{a}: \mathbf{X}^{(n)} \cup$ $\mathbf{A} \rightarrow \mathbf{Y}$ be an alteration of $f$ by $-c^{n}$, then by $(6.13) z\left(f_{a}\right)=z(f)-\delta c^{n}=0$. Hence $f_{a}$ may be extended to a map $g: \mathbf{X}^{(n+1)} \cup \mathbf{A} \rightarrow \mathbf{Y}$, and

$$
\begin{aligned}
g \mid \mathbf{X}^{(n-1)} \cup \mathbf{A} & =f_{a} \mid \mathbf{X}^{(n-1)} \cup \mathbf{A} \\
& =f \mid \mathbf{X}^{(n-1)} \cup \mathbf{A} .
\end{aligned}
$$

Conversely, suppose such a map $g$ exists. Let $f^{\prime}=g \mid \mathbf{X}^{(n)} \cup \mathbf{A}$. Then $z\left(f^{\prime}\right)=0$ and $f^{\prime}\left|\mathbf{X}^{(n-1)} \cup \mathbf{A}=f\right| \mathbf{X}^{(n-1)} \cup \mathbf{A}$ so by $(6.10) h(f)=h\left(f^{\prime}\right)=$ 0 .

Definition 6.15. Obstruction to a homotopy. Suppose $f_{0}, f_{1}: \mathbf{X} \rightarrow \mathbf{Y}$, and let $K:\left(\mathbf{A} \times \mathbf{I}, x_{0} \times \mathbf{I}\right) \rightarrow\left(\mathbf{Y}, y_{0}\right)$ be a homotopy $f_{0}\left|\mathbf{A} \cong f_{1}\right| \mathbf{A}\left(\right.$ rel $\left.x_{0}\right)$. Let $\overline{\mathbf{A}}_{01}=\mathbf{X} \times(0) \cup \mathbf{A} \times \mathbf{I} \cup \mathbf{X} \times(1) \subset \mathbf{X} \times \mathbf{I}$. Define $H_{0}: \overline{\mathbf{A}}_{01} \rightarrow \mathbf{Y}$ by

$$
H_{0}(x, \sigma)= \begin{cases}f_{0}(x) & \text { if }(x, \sigma) \in \mathbf{X} \times(0) \\ K(x, \sigma) & \text { if }(x, \sigma) \in \mathbf{A} \times \mathbf{I} \\ f_{1}(x) & \text { if }(x, \sigma) \in \mathbf{X} \times(1)\end{cases}
$$

Since $H\left(x_{0} \times \mathbf{I}\right)=\left\{y_{0}\right\}$ we may think of $H$ as a map $\overline{\mathbf{A}}_{01} / x_{0} \times \mathbf{I} \rightarrow \mathbf{Y}$, and apply obstruction theory to the one pointed pair $\left(\mathbf{X} \times \mathbf{I} / x_{0} \times \mathbf{I}, \overline{\mathbf{A}}_{01} / x_{0} \times \mathbf{I}\right)$. Furthermore the obstruction classes are in the cohomology groups

$$
\begin{aligned}
H^{n+1}\left(\mathbf{X} \times \mathbf{I}, \overline{\mathbf{A}}_{01} ; \pi_{n}(\mathbf{W})_{\phi \theta}\right) & =H^{n+1}\left(\Sigma \mathbf{X}, \Sigma \mathbf{A} ; \pi_{n}(\mathbf{W})_{\phi \theta}\right) \\
& \cong H^{n}\left(\mathbf{X}, \mathbf{A} ; \pi_{n}(\mathbf{W})_{\phi \theta}\right)
\end{aligned}
$$

Corresponding to (6.4) we have the following:

Lemma 6.16. $\quad H_{0}$ extends to $H_{2}:(\mathbf{X} \times \mathbf{I})^{(2)} \cup \overline{\mathbf{A}}_{01} \rightarrow \mathbf{Y}$ if and only if $f_{0}$ and $f_{1}$ induce the same homomorphism $\theta: \pi_{1}(\mathbf{X}) \rightarrow \pi_{1}(\mathbf{Y})$.

Proof. Suppose such an $H_{2}$ exists. Let $i: \overline{\mathbf{A}}_{01} \rightarrow \mathbf{X} \times \mathbf{I}$ be inclusion, and $i_{0}: \mathbf{X} \rightarrow \mathbf{X} \times(0) \subset \overline{\mathbf{A}}_{01}, i_{1}: \mathbf{X} \rightarrow \mathbf{X} \times(1) \subset \overline{\mathbf{A}}_{01}$. then $i \circ i_{0}$ and $i \circ i_{1}$ are 
homotopic maps $\mathbf{X} \rightarrow \mathbf{X} \times \mathbf{I}$. We have

$$
\begin{aligned}
f_{0 *} & =H_{2 *} i_{*} i_{0 *} \\
& =H_{2 *} i_{*} i_{1 *} \\
& =f_{1 *} .
\end{aligned}
$$

Conversely, suppose $f_{0}$ and $f_{1}$ both induce $\theta: \pi_{1}(\mathbf{X}) \rightarrow \pi_{1}(\mathbf{Y})$. Let $\chi: \pi_{1}(\mathbf{X}) \rightarrow \pi_{1}(\mathbf{X} \times \mathbf{I})$ be the isomorphism defined by $\chi=i_{*} i_{0 *}=i_{*} i_{1 *}$. Let $\bar{\theta}: \pi_{1}(\mathbf{X} \times \mathbf{I}) \rightarrow \pi_{1}(\mathbf{Y})$ be given by $\bar{\theta}=\theta \circ \chi^{-1}$. We claim that $H_{0 *}=\bar{\theta} i_{*}$. Therefore, by (6.4) the extension $H_{2}$ exists.

An application of the Van Kampen theorem shows that the following diagram is a pushout

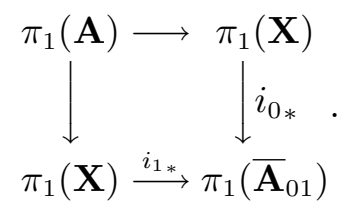

Hence any $\alpha \in \pi_{1}\left(\overline{\mathbf{A}}_{01}\right)$ can be written as a product:

$$
\alpha=i_{0 *}\left(x_{1}\right) i_{1 *}\left(x_{2}\right) \ldots i_{0 *}\left(x_{2 k-1}\right) i_{1 *}\left(x_{2 k}\right)
$$

where $x_{i} \in \pi_{1}(\mathbf{X})$, for all $i$. It follows that:

$$
\begin{aligned}
H_{0 *}(\alpha) & =H_{0 *} i_{0 *}\left(x_{1}\right) H_{0 *} i_{1 *}\left(x_{2}\right) \ldots \\
& =f_{0 *}\left(x_{1}\right) f_{1 *}\left(x_{2}\right) \ldots \\
& =\theta\left(x_{1}\right) \theta\left(x_{2}\right) \ldots \\
& =\theta\left(x_{1} x_{2} \ldots\right) .
\end{aligned}
$$

On the other hand

$$
\begin{aligned}
\bar{\theta} \circ i_{*}(\alpha) & =\theta \chi^{-1}\left(i_{*} i_{0 *}\left(x_{1}\right) i_{*} i_{1 *}\left(x_{2}\right) \ldots\right) \\
& =\theta \chi^{-1}\left(\chi\left(x_{1}\right) \chi\left(x_{2}\right) \ldots\right) \\
& =\theta\left(x_{1} x_{2} \ldots\right)
\end{aligned}
$$

Thus $H_{0 *}=\bar{\theta} i_{*}$.

\section{Semi-Localization.}

Let $P$ be a finite collection of prime integers, and let $\mathbf{Z}_{(P)}$ be the integers localized with respect to $P$. We show that if $\mathbf{X}$ is a one pointed Kan complex, there exists a diagram $\mathbf{X} \stackrel{f}{\rightarrow} \mathbf{X}_{p}$ such that $f_{*}: \pi_{1}(\mathbf{X}) \rightarrow \pi_{1}\left(X_{p}\right)$ is an 
isomorphism, and $f_{*} \otimes \mathbf{Z}_{(P)}: \pi_{k}(\mathbf{X}) \rightarrow \pi_{k}\left(\mathbf{X}_{p}\right)$ is an isomorphism for $k \geq 2$. This diagram is characterized by a universal mapping property.

Remark 7.1. Note that if $f: \mathbf{X} \rightarrow \mathbf{Y}$ is a semi-simplicial map, then there exits a factorization:

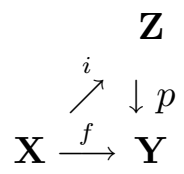

such that $i$ is an inclusion, and $p$ is a fibration. Furthermore the factorization can be chosen so that $i$ or $p$ is a weak homotopy equivalence, as preferred. If in addition $\mathbf{X}$ and $\mathbf{Y}$ are Kan complexes, then, since $p$ is a fibration, $\mathbf{Z}$ must also be a Kan Complex. If $\mathbf{X}$ and $\mathbf{Y}$ are connected then $\mathbf{Z}$ must also be connected.

If $\mathbf{Z}$ is a connected Kan complex, and $z_{0} \in Z_{0}$, define a one pointed subcomplex $\mathbf{Z}^{\prime} \leq \mathbf{Z}$ as follows. Let $Z_{0}^{\prime}=\left\{z_{0}\right\}$. Inductively for $q \geq 1$ let $Z_{q}^{\prime}=\left\{z \in Z_{q} \mid \partial_{i} z \in Z_{q-1}^{\prime}, \quad 0 \leq i \leq q\right\}$. It is straightforward to check that $\mathbf{Z}^{\prime}$ is a Kan complex, and the inclusion $\mathbf{Z}^{\prime} \leq \mathbf{Z}$ is a homotopy equivalence. It follows that if $f: \mathbf{X} \rightarrow \mathbf{Y}$ is a map of one pointed Kan complexes then there is a factorization:

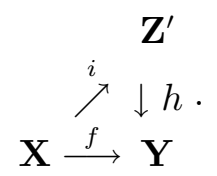

Such that $i$ is an inclusion, $\mathbf{Z}^{\prime}$ is a one pointed Kan complex, and $h$ is a homotopy equivalence.

Any Kan fiber sequence $(\mathbf{Z}, p, \mathbf{Y})$ contains a minimal sub fiber sequence $\left(\mathbf{Z}^{\prime}, p^{\prime}, \mathbf{Y}^{\prime}\right)$ as a deformation retraction. It follows that there is a commuting diagram:

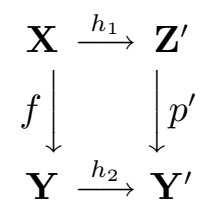

such that $h_{1}$, and $h_{2}$ are homotopy equivalences, and $\left(\mathbf{Z}^{\prime}, p^{\prime}, \mathbf{Y}^{\prime}\right)$ is a minimal fiber sequence.

Lemma 7.2. Let $\mathbf{F} \stackrel{i}{\rightarrow} \mathbf{E} \stackrel{p}{\rightarrow} \mathbf{B}$ be a Kan fiber sequence of connected Kan complexes, $(G, \phi)$ be a $\pi_{1}(\mathbf{B})$-module, and suppose

$$
H^{q}(\mathbf{F} ; G)= \begin{cases}G & \text { if } q=0 \\ 0 & \text { if } q>0\end{cases}
$$


Then $p^{*}: H^{q}\left(\mathbf{B} ; G_{\phi}\right) \rightarrow H^{q}\left(\mathbf{E} ; G_{\phi p_{*}}\right)$ is an isomorphism for all $q$.

Proof. Consider the spectral sequence of (4.2). We have a filtration preserving map of fibrations

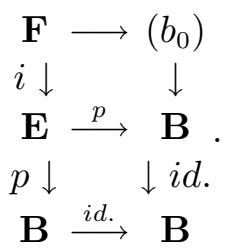

Let $\left\{E_{r}, d_{r}\right\}$ be the spectral sequence corresponding to $p$, and let $\left\{E_{r}^{\prime}, d_{r}^{\prime}\right\}$ be the spectral sequence corresponding to $i d$. Notice that $p$ induces an isomorphism on the $2^{\text {nd }}$ terms of the spectral sequences, since

$$
\begin{aligned}
E_{2}^{p, q} & =H^{p}\left(\mathbf{B} ; H^{q}(\mathbf{F} ; G)_{\tilde{\phi}}\right) \\
& = \begin{cases}H^{p}\left(\mathbf{B} ; G_{\phi}\right) & \text { if } q=0 ; \\
0 & \text { if } q>0 ;\end{cases} \\
& =E_{2}^{\prime p, q} .
\end{aligned}
$$

But the filtration is finite in each degree, so this implies the result, see $[3]$.

Definition 7.3. A Kan complex $\mathbf{Y}$ such that $\pi_{k}(\mathbf{Y})$ is a $\mathbf{Z}_{(P)}$-module for all $k \geq 2$ is called a semi- $\mathbf{Z}_{(P)}$ complex.

Definition 7.4. Let $\mathbf{X}$ be a one pointed Kan complex, with $\pi=\pi_{1}(\mathbf{X})$. View $\mathbf{X}$ as a complex over $\overline{\mathbf{W}}(\pi)$ by letting $\hat{x}: \mathbf{X} \rightarrow \overline{\mathbf{W}}(\pi)$ be the unique map inducing the identity homomorphism on fundamental groups, see (3.12). Then $\mathbf{X}$ will be called a $\pi$ complex.

Definition 7.5. A $\pi$ complex which is also a semi- $\mathbf{Z}_{(P)}$ complex will be called a $\pi$-semi-Z $\mathbf{Z}_{(P)}$ complex.

Lemma 7.6.

(i) Let $\mathbf{X}$ and $\mathbf{Y}$ be $\pi$-complexes, and $f: \mathbf{X} \rightarrow \mathbf{Y}$ be a semi-simplicial map. Then $f$ is a map over $\overline{\mathbf{W}}(\pi)$ if and only if $f$ induces the identity homomorphism on fundamental groups.

(ii) If $f_{0}, f_{1}: \mathbf{X} \rightarrow \mathbf{Y}$ are maps over $\overline{\mathbf{W}}(\pi)$, and $H:\left(\mathbf{X} \times \mathbf{I}, x_{0} \times \mathbf{I}\right) \rightarrow$ $\left(\mathbf{Y}, y_{0}\right)$ is a homotopy $f_{0} \cong f_{1}$ (rel $\left.x_{0}\right)$ then $H$ is a homotopy over $\overline{\mathbf{W}}(\pi)$.

Proof. (i) If $f$ is a map over $\overline{\mathbf{W}}(\pi)$ then $\hat{y} \circ f=\hat{x}$, but $\hat{x}$ and $\hat{y}$ both induce the identity homomorphism on fundamental groups, therefore $f$ does too. 
Conversely if $f$ induces the identity homomorphism on fundamental groups, then so does $\hat{y} \circ f: \mathbf{X} \rightarrow \overline{\mathbf{W}}(\pi)$, but $\hat{x}$ is the unique map with this property so $\hat{y} \circ f=\hat{x}$.

(ii) Let $H:\left(\mathbf{X} \times \mathbf{I}, x_{0} \times \mathbf{I}\right) \rightarrow\left(\mathbf{Y}, y_{0}\right)$ be a homotopy $f_{0} \cong f_{1}\left(\right.$ rel $\left.x_{0}\right)$. The following diagram commutes:

$$
\begin{array}{ccc}
\pi_{1}\left(\mathbf{X} \times \mathbf{I} / x_{0} \times \mathbf{I}\right) \stackrel{H_{*}}{\longrightarrow} & \pi_{1}(\mathbf{Y}) \\
p_{1 *} \mid \uparrow i_{0 *} & \stackrel{f_{0 *}}{\downarrow} & \hat{y}_{*} \\
\pi_{1}(\mathbf{X}) & \stackrel{\hat{x}_{*}}{\longrightarrow} & \pi_{1}(\overline{\mathbf{W}}(\pi))
\end{array} .
$$

Then we have $\hat{y}_{*} H_{*}=\hat{x}_{*} p_{1 *}$. But now it follows from (3.12) that $\hat{y} \circ H=$ $\hat{x} \circ p_{1}$.

Theorem 7.7. Let $\mathbf{X}$ be a $\pi$ complex, $\mathbf{X}_{p}$ be a $\pi$-semi- $\mathbf{Z}_{(P)}$ complex, $f: \mathbf{X} \rightarrow \mathbf{X}_{p}$ be a map over $\overline{\mathbf{W}}(\pi)$ and $(G, \phi)$ be a $\pi$-module, where $G$ is also a $\mathbf{Z}_{(P)}$ module. If $f_{*} \otimes \mathbf{Z}_{(P)}: \pi_{k}(\mathbf{X}) \otimes \mathbf{Z}_{(P)} \rightarrow \pi_{k}\left(\mathbf{X}_{p}\right) \otimes \mathbf{Z}_{(P)}=\pi_{k}\left(\mathbf{X}_{p}\right)$ is an isomorphism for all $k \geq 2$, then $f^{*}: H^{q}\left(\mathbf{X}_{p} ; G_{\phi}\right) \rightarrow H^{q}\left(\mathbf{X} ; G_{\phi}\right)$ is an isomorphism for all $q \geq 0$.

Proof. By (7.1) there exists a diagram

$$
\begin{gathered}
\mathbf{X} \stackrel{h_{1}}{\cong} \mathbf{E} \\
f \downarrow \\
\mathbf{X}_{p} \stackrel{h_{2}}{\cong} \mathbf{B}
\end{gathered}
$$

where $(\mathbf{E}, p, \mathbf{B})$ is a minimal fiber space, and $h_{1}$ and $h_{2}$ are homotopy equivalences. Since $h_{1}$ and $h_{2}$ are homotopy equivalences, and $f$ induces the identity map on fundamental groups, $p_{*}: \pi_{1}(\mathbf{E}) \rightarrow \pi_{1}(\mathbf{B})$ is an isomorphism, and $p_{*} \otimes \mathbf{Z}_{(P)}: \pi_{k}(\mathbf{E}) \otimes \mathbf{Z}_{(P)} \rightarrow \pi_{k}(\mathbf{B}) \otimes \mathbf{Z}_{(P)}$ is an isomorphism for all $k \geq 2$. It will suffice to prove the result for $p$.

Let $\mathbf{F}$ be the fiber of $p$. $\mathbf{E}$ is connected, so we have the following exact sequence of pointed sets

$$
\pi_{1}(\mathbf{E}) \stackrel{p_{*}}{\cong} \pi_{1}(\mathbf{B}) \stackrel{\partial}{\rightarrow} \pi_{0}(\mathbf{F}) \stackrel{i_{*}}{\rightarrow} \pi_{0}(\mathbf{E})=0
$$

It follows that $\mathbf{F}$ is connected. We also have the exact sequence

$$
\ldots \rightarrow \pi_{2}(\mathbf{E}) \stackrel{p_{*}}{\rightarrow} \pi_{2}(\mathbf{B}) \stackrel{\partial}{\rightarrow} \pi_{1}(\mathbf{F}) \rightarrow 0 .
$$

Hence $\pi_{1}(\mathbf{F}) \cong \pi_{2}(\mathbf{B}) / p_{*} \pi_{2}(\mathbf{E})$, and we see that $\pi_{1}(\mathbf{F})$ is abelian. Tensoring the long exact homotopy sequence of the fibration with $\mathbf{Z}_{(P)}$ we obtain the result $\pi_{k}(\mathbf{F}) \otimes \mathbf{Z}_{(P)}=0, \quad k \geq 1$. 
To show that $p^{*}: H^{q}\left(\mathbf{B} ; G_{\phi h_{2}-1}\right) \rightarrow H^{q}\left(\mathbf{E} ; G_{\phi h_{1 *}^{-1}}\right)$ is an isomorphism for all $q$, by (7.2) it suffices to prove:

$$
H^{q}(\mathbf{F} ; G)= \begin{cases}G & \text { if } q=0 \\ 0 & \text { if } q>0\end{cases}
$$

By the universal coefficient theorem it suffices to prove:

$$
H_{q}\left(\mathbf{F} ; \mathbf{Z}_{(P)}\right)= \begin{cases}G & \text { if } q=0 \\ 0 & \text { if } q>0\end{cases}
$$

We conclude by proving the following lemma:

Lemma 7.8. Suppose $\pi_{k}(\mathbf{F}) \otimes \mathbf{Z}_{(P)}=0, k \geq 1$, then:

$$
H_{q}\left(\mathbf{F} ; \mathbf{Z}_{(P)}\right)= \begin{cases}\mathbf{Z}_{(P)} & \text { if } q=0 \\ 0 & \text { if } q>0 .\end{cases}
$$

Proof. (i) First we show that if $G \otimes \mathbf{Z}_{(P)}=0$ then

$$
H_{q}\left(\mathbf{K}(G, n) ; \mathbf{Z}_{(P)}\right)= \begin{cases}\mathbf{Z}_{(P)} & \text { if } q=0 \\ 0 & \text { if } q>0\end{cases}
$$

If $n \geq 2$ then this follows from [11]. Suppose $n=1$. If $G=\mathbf{Z} / m \mathbf{Z}$ then

$$
H_{q}(\mathbf{K}(G, 1) ; Z)= \begin{cases}G & \text { if } q \text { is odd; } \\ 0 & \text { if } q \geq 2 \text { is even; } \\ Z & \text { if } q=0\end{cases}
$$

But

$$
H_{q}\left(\mathbf{K}(G, 1) ; \mathbf{Z}_{(P)}\right)=H_{q}(\mathbf{K}(G, 1) ; Z) \otimes \mathbf{Z}_{(P)}
$$

so the result follows.

If $G$ is a finite sum of cyclic groups $\mathbf{Z} / m_{i} \mathbf{Z}$ then we must have $\mathbf{Z} / m_{i} \mathbf{Z} \otimes$ $\mathbf{Z}_{(P)}=0$ for all $i$. Furthermore $\mathbf{K}(G, 1)$ is a product of the complexes $\mathbf{K}\left(\mathbf{Z} / m_{i} \mathbf{Z}, 1\right)$, again the result follows.

In general $G$ is a direct limit of finite sums of cyclic groups. Since homology commutes with direct limits the result follows in general. 
(ii) We prove the result by induction on the Postnikov tower for $\mathbf{F}$. Let $\pi_{n}=\pi_{n}(\mathbf{F})$. Note that $\mathbf{F}_{1}=\mathbf{K}\left(\pi_{1}, 1\right)$ hence

$$
H_{q}\left(\mathbf{F}_{1} ; \mathbf{Z}_{(P)}\right)= \begin{cases}\mathbf{Z}_{(P)} & \text { if } q=0 \\ 0 & \text { if } q>0 .\end{cases}
$$

By the universal coefficient theorem:

$$
H^{q}\left(\mathbf{F}_{1} ; \mathbf{Z}_{(P)}\right)= \begin{cases}\mathbf{Z}_{(P)} & \text { if } q=0 \\ 0 & \text { if } q>0 .\end{cases}
$$

Inductively suppose that

$$
H^{q}\left(\mathbf{F}_{n-1} ; \mathbf{Z}_{(P)}\right)= \begin{cases}\mathbf{Z}_{(P)} & \text { if } q=0 \\ 0 & \text { if } q>0\end{cases}
$$

Consider the spectral sequence (4.2) for the fibration $\mathbf{K}\left(\pi_{n}, n\right) \stackrel{i}{\rightarrow} \mathbf{F}_{n} \stackrel{p}{\rightarrow} \mathbf{F}_{n-1}$ with ordinary coefficients in $\mathbf{Z}_{(P)}$. The second term of the sequence is given by

$$
\begin{aligned}
E_{2}^{p, q} & =H^{p}\left(\mathbf{F}_{n-1} ; H^{q}\left(\mathbf{K}\left(\pi_{n}, n\right) ; \mathbf{Z}_{(P)}\right)\right) \\
& =0 \quad \text { whenever }(p, q) \neq(0,0) .
\end{aligned}
$$

Whence

$$
H^{q}\left(\mathbf{F}_{n} ; \mathbf{Z}_{(P)}\right)= \begin{cases}\mathbf{Z}_{(P)} & \text { if } q=0 ; \\ 0 & \text { if } q>0 .\end{cases}
$$

But $\mathbf{F}$ is the projective limit of the $\mathbf{F}_{n}$ and cohomology commutes with projective limits, therefore

$$
H^{q}\left(\mathbf{F} ; \mathbf{Z}_{(P)}\right)= \begin{cases}\mathbf{Z}_{(P)} & \text { if } q=0 \\ 0 & \text { if } q>0 .\end{cases}
$$

Now the result follows by the universal coefficient theorem.

Definition 7.9. Let $\mathbf{X}$ be a $\pi$-complex, $\mathbf{X}_{p}$ be a $\pi$-semi- $\mathbf{Z}_{(P)}$ complex and $f: \mathbf{X} \rightarrow \mathbf{X}_{p}$ be a map over $\overline{\mathbf{W}}(\pi)$. Then $f$ is called a $P$ semi-localization if it has the following universal property with respect to maps of $\mathbf{X}$ into semi- $\mathbf{Z}_{(P)}$ complexes:

Let $g: \mathbf{X} \rightarrow \mathbf{Y}$ where $\mathbf{Y}$ is a semi- $\mathbf{Z}_{(P)}$ complex, then there exists $h$ : $\mathbf{X}_{p} \rightarrow \mathbf{Y}$ such that $h \circ f=g$. Furthermore If $g_{0}, g_{1}: \mathbf{X} \rightarrow \mathbf{Y}$ are homotopic $\left(\right.$ rel $\left.x_{0}\right)$, and if $h_{0}, h_{1}: \mathbf{X}_{p} \rightarrow \mathbf{Y}$ such that $h_{i} \circ f=g_{i}, i=0,1$, then $h_{0} \cong h_{1}$ $\left(\right.$ rel $\left.x_{0}^{\prime}\right)$. 
Remark 7.10. The following are consequences of (7.5).

(i) If $\mathbf{Y}$ is a $\pi$-semi- $\mathbf{Z}_{(P)}$ complex and $g$ is a map over $\overline{\mathbf{W}}(\pi)$ then so is $h$, because $h_{*} f_{*}=g_{*}$ on fundamental groups, and $f$ and $g$ are maps over $\overline{\mathbf{W}}(\pi)$ so $f$ and $g$ both induce the identity homomorphism on fundamental groups, so $h$ must induce the identity homomorphism on fundamental groups. Hence $h$ is a map over $\overline{\mathbf{W}}(\pi)$.

(ii) If $g_{0}, g_{1}, h_{0}, h_{1}$ are all maps over $\overline{\mathbf{W}}(\pi)$ with $g_{0} \cong g_{1}\left(\right.$ rel $\left.x_{0}\right)$ over $\overline{\mathbf{W}}(\pi)$, and $h_{i} \circ f=g_{i}, i=0,1$. Then $h_{0} \cong h_{1}\left(\right.$ rel $\left.x_{0}^{\prime}\right)$ over $\overline{\mathbf{W}}(\pi)$ since all based homotopies of maps over $\overline{\mathbf{W}}(\pi)$ are homotopies over $\overline{\mathbf{W}}(\pi)$.

Proposition 7.11. P-semi-localization is unique up to homotopy equivalence.

Proof. This is proved in the usual manner from the universal property.

Theorem 7.12. Let $\mathbf{X}$ be a $\pi$ complex, $\mathbf{X}_{p}$ be a $\pi$-semi- $\mathbf{Z}_{(P)}$ complex and $f: \mathbf{X} \rightarrow \mathbf{X}_{p}$ be a map over $\overline{\mathbf{W}}(\pi)$. If $f$ has the property that whenever $G$ is a $\mathbf{Z}_{(P)}$ module, then $f^{*}: H^{q}\left(\mathbf{X}_{p} ; G_{\phi}\right) \rightarrow H^{q}\left(\mathbf{X} ; G_{\phi}\right)$ is an isomorphism for all $q \geq 0$, then $f$ is the P-semi-localization.

Proof. By (7.1), up to homotopy equivalence, we may assume $f$ is an inclusion. Let $g: \mathbf{X} \rightarrow \mathbf{Y}$ where $\mathbf{Y}$ is a semi- $\mathbf{Z}_{(P)}$ complex. Since $f$ is a map over $\overline{\mathbf{W}}(\pi)$ it induces the identity homomorphism on fundamental groups, so the following diagram commutes:

$$
\begin{aligned}
\pi_{1}(\mathbf{X}) \stackrel{f_{*}}{\cong} & \pi_{1}\left(\mathbf{X}_{p}\right) \\
g_{*} \searrow & \downarrow \theta \\
& \pi_{1}(\mathbf{Y})
\end{aligned} .
$$

Where $\theta=g_{*}$. Thus $g$ extends to $h_{2}: \mathbf{X}_{p}^{(2)} \cup \mathbf{X} \rightarrow \mathbf{Y}$ by (6.4).

Inductively suppose $n \geq 3$, and $g$ has an extension $h_{n-1}: \mathbf{X}_{p}^{(n-1)} \cup \mathbf{X} \rightarrow \mathbf{Y}$, then the obstruction to extending $g$ over $\mathbf{X}_{p}^{(n)} \cup \mathbf{X}$ lies in $H^{n}\left(\mathbf{X}_{p}, \mathbf{X} ; \pi_{n-1}(\mathbf{Y})_{\theta}\right)$. But this group vanishes by the long exact sequence for the pair, because $\pi_{n-1}(\mathbf{Y})$ is a $\mathbf{Z}_{(P)}$ module, hence $f^{*}: H^{q}\left(\mathbf{X}_{p} ; \pi_{n-1}(\mathbf{Y})_{\theta}\right) \rightarrow H^{q}\left(\mathbf{X} ; \pi_{n-1}(\mathbf{Y})_{\theta}\right)$ is an isomorphism for all $q \geq 0$ by hypothesis. Hence $g$ has an extension $h: \mathbf{X}_{p} \rightarrow \mathbf{Y}$ such that $h \circ f=g$.

Suppose $g_{0}, g_{1}: \mathbf{X} \rightarrow \mathbf{Y}$ such that $g_{0} \cong g_{1}\left(\right.$ rel $\left.x_{0}\right)$, and that $h_{0}, h_{1}:$ $\mathbf{X}_{p} \rightarrow \mathbf{Y}$ with $h_{i} \circ f=g_{i}, i=0,1$. The homomorphisms induced on the fundamental groups are

$$
\begin{aligned}
h_{0 *} & =g_{0 *} f_{*}^{-1} \\
& =g_{1 *} f_{*}^{-1} \\
& =h_{1 *} .
\end{aligned}
$$


Thus $h_{0}\left|\mathbf{X}_{p}^{(1)} \cup \mathbf{X} \cong h_{1}\right| \mathbf{X}_{p}^{(1)} \cup \mathbf{X} \quad\left(\right.$ rel $\left.x_{0}\right)$ by $(6.16)$.

Inductively suppose $n \geq 2$ and $h_{0}\left|\mathbf{X}_{p}^{(n-1)} \cup \mathbf{X} \cong h_{1}\right| \mathbf{X}_{p}^{(n-1)} \cup \mathbf{X}\left(\right.$ rel $\left.x_{0}\right)$. The obstruction to a homotopy $\left.h_{0}\left|\mathbf{X}_{p}^{(n)} \cup \mathbf{X} \cong h_{1}\right| \mathbf{X}_{p}^{(} n\right) \cup \mathbf{X}\left(\right.$ rel $\left.x_{0}\right)$ lies in $H^{n}\left(\mathbf{X}_{p}, \mathbf{X} ; \pi_{n}(\mathbf{Y})_{\theta}\right)=0$ as above. Hence $h_{0} \cong h_{1}\left(\right.$ rel $\left.x_{0}\right)$.

Note that (7.7) and (7.12) imply that if $f: \mathbf{X} \rightarrow \mathbf{X}_{p}$ is an inclusion satisfying $f_{*} \otimes \mathbf{Z}_{(P)}: \pi_{k}(\mathbf{X}) \otimes \mathbf{Z}_{(P)} \rightarrow \pi_{k}\left(\mathbf{X}_{p}\right)$ is an isomorphism for all $k \geq 2$, then $f$ is the $\mathrm{P}$-semi-localization.

Theorem 7.13. Let $\mathbf{X}$ be a one pointed Kan complex, then $\mathbf{X}$ has a Psemi-localization.

Proof. We construct the $P$-semi-localization by induction on the Postnikov tower of $\mathbf{X}$. Let $\pi=\pi_{1}(\mathbf{X}), \pi_{n}=\pi_{n}(\mathbf{X})$. Let $\mathbf{X}_{n}$ be the $n^{\text {th }}$ stage of the Postnikov system for $\mathbf{X}$.

$\mathbf{X}_{1}$ is a one pointed Kan complex of type $(\pi, 1)$. It follows that if $f_{1}$ : $\mathbf{X}_{1} \rightarrow \overline{\mathbf{W}}(\pi)$ is the unique map inducing the identity homomorphism on fundamental groups then $f_{1}$ is a P-semi-localization.

Suppose inductively that we have a P-semi-localization $f_{n-1}: \mathbf{X}_{n-1} \rightarrow$ $\mathbf{X}_{n-1, p}$. Then by the universal property there exists a unique map, up to homotopy, $k_{p}^{n}: \mathbf{X}_{n-1, p} \rightarrow \mathbf{L}_{\pi}\left(\pi_{n} \otimes \mathbf{Z}_{(P)}, n+1\right)$ over $\overline{\mathbf{W}}(\pi)$, such that the following diagram commutes

$$
\begin{gathered}
\mathbf{X}_{n-1} \stackrel{k^{n}}{\rightarrow} \quad \mathbf{L}_{\pi}\left(\pi_{n}, n+1\right) \\
f_{n-1} \downarrow \\
\mathbf{X}_{n-1, p} \stackrel{k_{p}^{n}}{\rightarrow} \mathbf{L}_{\pi}\left(\pi_{n} \otimes \mathbf{Z}_{(P)}, n+1\right)
\end{gathered}
$$

where $k^{n}$ is the $n^{\text {th }} k$-invariant. Let the following be pullback over $\overline{\mathbf{W}}(\pi)$

$$
\begin{gathered}
\mathbf{X}_{n, p} \rightarrow \mathbf{P}_{\pi}\left(\pi_{n} \otimes \mathbf{Z}_{(P)}, n+1\right) \\
q_{n} \downarrow \\
\mathbf{X}_{n-1, p} \stackrel{k_{p}^{n}}{\rightarrow} \mathbf{L}_{\pi}\left(\pi_{n} \otimes \mathbf{Z}_{(P)}, n+1\right)
\end{gathered} .
$$

We deduce from the long exact homotopy sequence for the fiber sequence

$$
\mathbf{K}\left(\pi_{n} \otimes \mathbf{Z}_{(P)}, n\right) \stackrel{i}{\rightarrow} \mathbf{X}_{n, p} \stackrel{q_{n}}{\rightarrow} \mathbf{X}_{n-1, p}
$$

that $\pi_{n}\left(\mathbf{X}_{n, p}\right)=\pi_{n} \otimes \mathbf{Z}_{(P)}$, and $q_{n_{*}}: \pi_{k}\left(\mathbf{X}_{n, p}\right) \rightarrow \pi_{k}\left(\mathbf{X}_{n-1, p}\right)$ is an isomorphism for $k \neq n$.

By the universal property of the pullback there exists a map $f_{n}: \mathbf{X}_{n} \rightarrow$ 
$\mathbf{X}_{n, p}$ rendering the following diagram commutative

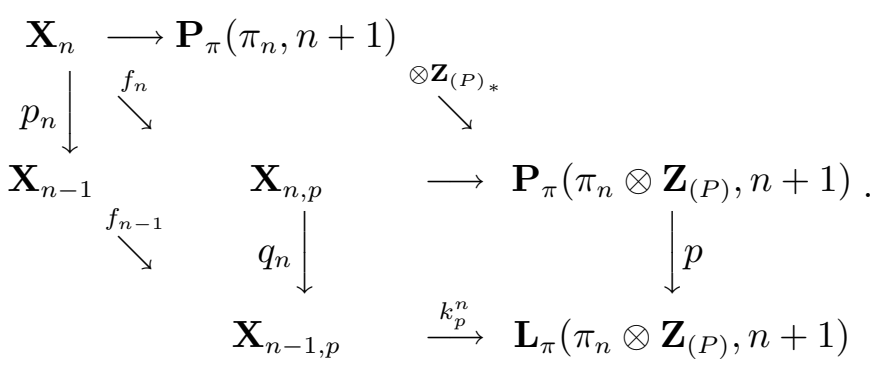

Note that:

$$
\begin{aligned}
\mathbf{X}_{n} & \subset \mathbf{P}_{\pi}\left(\pi_{n}, n+1\right) \times_{\overline{\mathbf{w}}(\pi)} \mathbf{X}_{n-1} ; \\
\mathbf{X}_{n, p} & \subset \mathbf{P}_{\pi}\left(\pi_{n} \otimes \mathbf{Z}_{(P)}, n+1\right) \times_{\overline{\mathbf{W}}(\pi)} \mathbf{X}_{n-1, p} \\
f_{n}((\mu, x), \sigma) & =\left(\left(\mu \otimes \mathbf{Z}_{(P)}, x\right), f_{n-1}(\sigma)\right) .
\end{aligned}
$$

Claim. $f_{n_{*}}: \pi_{k}\left(\mathbf{X}_{n}\right) \otimes \mathbf{Z}_{(P)} \rightarrow \pi_{k}\left(\mathbf{X}_{n, p}\right) \otimes \mathbf{Z}_{(P)}$ is an isomorphism for all $k \geq 2$.

Proof. Note that the following diagram commutes for all $k \geq 2$

$$
\begin{array}{cc}
\pi_{k}\left(\mathbf{X}_{n}\right) \otimes \mathbf{Z}_{(P)} \stackrel{f_{n_{*} \otimes \mathbf{Z}_{(P)}}^{\longrightarrow}}{\longrightarrow} \pi_{k}\left(\mathbf{X}_{n, p}\right) \otimes \mathbf{Z}_{(P)} \\
p_{n_{*}} \otimes \mathbf{Z}_{(P)} \downarrow \\
\pi_{k}\left(\mathbf{X}_{n-1}\right) \otimes \mathbf{Z}_{(P)} \stackrel{\downarrow n_{*} \otimes \mathbf{Z}_{(P)}}{\stackrel{f_{n-w_{*} \otimes \mathbf{Z}_{(P)}}}{\longrightarrow}} \pi_{k}\left(\mathbf{X}_{n-1, p}\right) \otimes \mathbf{Z}_{(P)}
\end{array}
$$

Furthermore $f_{n-1_{*}} \otimes \mathbf{Z}_{(P)}$ is an isomorphism for all $k \geq 2$, while $p_{n_{*}} \otimes$ $\mathbf{Z}_{(P)}$, and $q_{n_{*}} \otimes \mathbf{Z}_{(P)}$ are isomorphisms for $k \neq n$. Hence $f_{n_{*}} \otimes \mathbf{Z}_{(P)}$ is an isomorphism for $k \neq n$.

Note that the following diagram commutes

$$
\begin{aligned}
& \mathbf{K}\left(\pi_{n}, n\right) \quad \stackrel{i}{\rightarrow} \mathbf{X}_{n} \stackrel{p_{n}}{\rightarrow} \mathbf{X}_{n-1} \\
& \otimes \mathbf{Z}_{(P)_{*} \downarrow} \downarrow \quad f_{n} \downarrow \quad f_{n-1} \downarrow . \\
& \mathbf{K}\left(\pi_{n} \otimes \mathbf{Z}_{(P)}, n\right) \stackrel{i}{\rightarrow} \mathbf{X}_{n, p} \stackrel{q_{n}}{\rightarrow} \mathbf{X}_{n-1, p}
\end{aligned}
$$

We obtain the following commutative diagram

$$
\begin{aligned}
& 0 \rightarrow \quad \pi_{n}\left(\mathbf{K}\left(\pi_{n}, n\right)\right) \otimes \mathbf{Z}_{(P)} \quad \stackrel{i_{*} \otimes \mathbf{Z}_{(P)}}{\cong} \pi_{n}\left(\mathbf{X}_{n}\right) \otimes \mathbf{Z}_{(P)} \rightarrow 0 \\
& \downarrow \cong \otimes \mathbf{Z}_{(P)_{*}} \quad f_{n_{*}} \otimes \mathbf{Z}_{(P)} \downarrow \\
& 0 \rightarrow \pi_{n}\left(\mathbf{K}\left(\pi_{n} \otimes \mathbf{Z}_{(P)}, n\right)\right) \otimes \mathbf{Z}_{(P)} \stackrel{i_{*} \otimes \mathbf{Z}_{(P)}}{\cong} \pi_{n}\left(\mathbf{X}_{n, p}\right) \otimes \mathbf{Z}_{(P)} \rightarrow 0
\end{aligned}
$$


The claim now follows. Hence $f_{n}: \mathbf{X}_{n} \rightarrow \mathbf{X}_{n, p}$ is the $P$-semi-localization.

The composite map over $\overline{\mathbf{W}}(\pi), \mathbf{X} \stackrel{p}{\rightarrow} \mathbf{X}_{n} \stackrel{f_{n}}{\rightarrow} \mathbf{X}_{n, p}$ induces isomorphisms $\pi_{k}(\mathbf{X}) \otimes \mathbf{Z}_{(P)} \cong \pi_{k}\left(\mathbf{X}_{n, p}\right), \quad 2 \leq k \leq n$. If we let $\mathbf{X}_{p}$ be the projective limit $\mathbf{X}_{p}=\lim _{\leftarrow_{n}} \mathbf{X}_{n, p}$. We obtain a map $f: \mathbf{X} \rightarrow \mathbf{X}_{p}$ over $\overline{\mathbf{W}}(\pi)$ such that $f_{*} \otimes \mathbf{Z}_{(P)}: \pi_{k}(\mathbf{X}) \otimes \mathbf{Z}_{(P)} \rightarrow \pi_{k}\left(\mathbf{X}_{p}\right)$ is an isomorphism for all $k \geq 2$.

Lemma 7.14. Let $\mathbf{X}$ be a $\pi$ complex, and $f^{\prime}: \mathbf{X} \rightarrow \mathbf{X}_{p}^{\prime}$ be an arbitrary P-semi-localization. Then $f_{*}^{\prime} \otimes \mathbf{Z}_{(P)}: \pi_{k}(\mathbf{X}) \otimes \mathbf{Z}_{(P)} \rightarrow \pi_{k}\left(\mathbf{X}_{p}\right)$ is an isomorphism for all $k \geq 2$.

Proof. By (7.13) there exists a $P$-semi-localization $f: \mathbf{X} \rightarrow \mathbf{X}_{p}$ such that $f_{*} \otimes \mathbf{Z}_{(P)}: \pi_{k}(\mathbf{X}) \otimes \mathbf{Z}_{(P)} \rightarrow \pi_{k}\left(\mathbf{X}_{p}\right)$ is an isomorphism for all $k \geq 2$. By (7.11) there exists a commutative diagram

$$
\begin{aligned}
& \mathbf{X} \stackrel{f^{\prime}}{\rightarrow} \mathbf{X}_{p}^{\prime} \\
& f \downarrow \nearrow h \\
& \mathbf{X}_{p}
\end{aligned}
$$

where $h$ is a homotopy equivalence. It follows that $f_{*}^{\prime} \otimes \mathbf{Z}_{(P)}$ is an isomorphism for all $k \geq 2$.

We have now proved our main result

Theorem 7.15. Let $\mathbf{X}$ be a $\pi$ complex, $\mathbf{X}_{p}$ be a $\pi$-semi- $\mathbf{Z}_{(P)}$ complex and $f: \mathbf{X} \rightarrow \mathbf{X}_{p}$ be a map over $\overline{\mathbf{W}}(\pi)$. Then the following are equivalent:

(i) $f_{*} \otimes \mathbf{Z}_{(P)}: \pi_{k}(\mathbf{X}) \otimes \mathbf{Z}_{(P)} \rightarrow \pi_{k}\left(\mathbf{X}_{p}\right)$ is an isomorphism for $k \geq 2$.

(ii) $f^{*}: H^{q}\left(\mathbf{X}_{p} ; G_{\phi}\right) \rightarrow H^{q}\left(\mathbf{X} ; G_{\phi}\right)$, is an isomorphism for all $q \geq 0$, whenever $G$ is a $\mathbf{Z}_{(P)}$-module.

(iii) $f$ has the universal prorerty for maps of $\mathbf{X}$ into semi- $\mathbf{Z}_{(P)}$ complexes.

Proof. (i) $\Rightarrow$ (ii). This is (7.7).

(ii) $\Rightarrow$ (iii). This is (7.12).

(iii) $\Rightarrow(\mathrm{i})$. This is $(7.14)$.

In the following $\mathbf{T}$ denotes the Milnor geometric realization functor.

Theorem 7.16. Suppose $f: \mathbf{X} \rightarrow \mathbf{X}_{p}$ has the universal property, Then $\mathbf{T}(f): \mathbf{T}(\mathbf{X}) \rightarrow \mathbf{T}\left(\mathbf{X}_{p}\right)$ also has the universal property.

Proof. Let $\mathbf{Y}$ be a topological space with $\pi_{k}(\mathbf{Y})$ a $\mathbf{Z}_{(P)}$ module for $k \geq 2$. Let $g: \mathbf{T}(\mathbf{X}) \rightarrow \mathbf{Y}$ be a continuous map. Let $\psi(g): \mathbf{X} \rightarrow \mathbf{S}(\mathbf{Y})$ be the 
adjoint map, see $[8,16.1]$. Then there exists a factorization:

$$
\begin{aligned}
& \mathbf{X} \stackrel{\psi(g)}{\rightarrow} \mathbf{S}(\mathbf{Y}) \\
& f \downarrow \nearrow h \\
& \mathbf{X}_{p}
\end{aligned} .
$$

Let $\phi(h \circ f): \mathbf{T}(\mathbf{X}) \rightarrow \mathbf{Y}$ be the adjoint map. Notice that $\phi(h \circ f)=$ $\phi(h) \circ \mathbf{T}(f)$. Furthermore $\phi(\psi(g))=g$, and we have the factorization:

$$
\begin{aligned}
& \mathbf{T}(\mathbf{X}) \quad \stackrel{g}{\rightarrow} \quad \mathbf{Y} \\
& T(f) \downarrow \nearrow \phi(h) \\
& \mathbf{T}\left(\mathbf{X}_{p}\right)
\end{aligned}
$$

Now suppose $g_{0}, g_{1}: \mathbf{T}(\mathbf{X}) \rightarrow \mathbf{Y}$ are homotopic (rel $x_{0}$ ) and suppose $h_{0}, h_{1}: \mathbf{T}\left(\mathbf{X}_{p}\right) \rightarrow \mathbf{Y}$ such that $h_{i} \circ \mathbf{T}(f)=g_{i}, i=0,1$. Let $H: \mathbf{T}(\mathbf{X} \times \mathbf{I}) \rightarrow$ $\mathbf{Y}$ be a homotopy $g_{0} \cong g_{1} \quad\left(\operatorname{rel} x_{0}\right)$. Then $\psi(H): \mathbf{X} \times \mathbf{I} \rightarrow \mathbf{S}(\mathbf{Y})$ is a homotopy $\psi\left(g_{0}\right) \cong \psi\left(g_{1}\right)\left(\right.$ rel $\left.x_{0}\right)$. Furthermore $\psi\left(h_{i} \circ \mathbf{T}(f)\right)=\psi\left(h_{i}\right) \circ f$. Thus we observe that $\psi\left(h_{i}\right) \circ f=\psi\left(g_{i}\right), \quad i=0,1$. Since $f$ has the universal property there exists a homotopy $H: \mathbf{X}_{p} \times \mathbf{I} \rightarrow \mathbf{S}(\mathbf{Y}), \quad \psi\left(h_{0}\right) \cong \psi\left(h_{1}\right)$ $\left(\right.$ rel $\left.x_{0}\right)$. But then the adjoint map $\phi(H): \mathbf{T}\left(\mathbf{X}_{P} \times \mathbf{I}\right) \rightarrow \mathbf{Y}$ is a homotopy $h_{0} \cong h_{1},\left(\right.$ rel $\left.x_{0}\right)$.

Example 7.17. Let $P$ be the empty set, in which case $\mathbf{Z}_{(P)}=\mathbf{Z}_{(0)}=\mathbf{Q}$, the rational numbers. Let $\mathbf{S}=\mathbf{S}^{2 n+1}$ be an odd dimensional sphere, $\pi$ be a finite group, and suppose $\mathbf{S}$ has a C.W. complex structure such that $\pi$ acts freely, cellularly on $\mathbf{S}$. Let $\mathbf{X}=\frac{\mathbf{S}}{\pi}$. $\mathbf{S}$ is the universal cover of $\mathbf{X}$, and $\pi_{1}(\mathbf{X})=\pi$. Let $\mathbf{M} \stackrel{i}{\rightarrow} \mathbf{S}(\mathbf{X})$ be the minimal subcomplex of the total singular complex of $\mathbf{X}$. Let $f: \mathbf{M} \rightarrow \mathbf{M}_{(0)}$ be the semi-localization. Note that

$$
\pi_{k}(\mathbf{S}) \otimes \mathbf{Q}= \begin{cases}\mathbf{Q} & \text { if } k=2 n+1 \\ \mathbf{0} & \text { if } k \neq 2 n+1\end{cases}
$$

Therefore

$$
\pi_{k}\left(\mathbf{M}_{(0)}\right)= \begin{cases}\pi & \text { if } k=1 \\ \mathbf{Q} & \text { if } k=2 n+1 \\ 0 & \text { if otherwise. }\end{cases}
$$

It follows that the $2 n^{\text {th }}$-stage of the Postnikov system of $\mathbf{M}_{(0)}$ looks like

$$
\begin{array}{ll} 
& \left(\mathbf{M}_{(0)}\right)^{(2 n+1)} \\
p_{2 n+1}^{\infty} \nearrow & \mid p_{2 n}^{2 n+1} \\
\mathbf{M}_{(0)} \rightarrow & \overline{\mathbf{W}}(\pi)
\end{array} .
$$


Where $p_{2 n+1}^{\infty}$ is a homotopy equivalence. Furthermore the $k$ invariant is an element of $H^{2 n+2}\left(\pi ; \mathbf{Q}_{\phi \otimes Q}\right)$. But this group is trivial because $\pi$ is finite. It follows that $\mathbf{M}_{(0)}$ is homotopy equivalent to an $\mathbf{L}_{\pi}(\mathbf{Q}, 2 n+1)$.

\section{Appendix. The cohomology of $\mathbf{L}_{\pi}(G, n)$.}

The following is a generalization of a theorem of J. Siegel, see [12, 3.7]. Let $G_{i}$ be an abelian group, $\phi_{i}: \pi \rightarrow \operatorname{Aut}\left(G_{i}\right)$ be a homomorphism $i=1,2$. According to $[\mathbf{1 2}, 3.6]$, the twisted coefficient cohomology $H^{*}\left(\mathbf{L}_{\pi}\left(G_{1}, m\right) ; G_{2 \phi_{2}}\right)$ can be computed from the total complex associated to the double complex $\operatorname{Hom}_{\mathbf{Z}[\pi]}\left(\Pi_{*}, C^{*}\left(\mathbf{K}\left(G_{1}, m\right) ; G_{2}\right)\right)$ where $\Pi_{*} \rightarrow \mathbf{Z}$ is a free $\mathbf{Z}[\pi]$-resolution of $\mathbf{Z}$.

According to G. Bredon [1], we can choose a C.W. model for $\mathbf{K}\left(G_{1}, m\right)$ such that $C_{q}\left(\mathbf{K}\left(G_{1}, m\right)\right)$ is a free $\mathbf{Z}[\pi]$ - module for $q>0$. Consequently $C^{q}\left(\mathbf{K}\left(G_{1}, m\right) ; G_{2}\right)$ is relative injective for $q>0$, see [5].

We filter the total complex associated to $\operatorname{Hom}_{\mathbf{Z}[\pi]}\left(\Pi_{*}, C^{*}\left(\mathbf{K}\left(G_{1}, m\right) ; G_{2}\right)\right)$ by the second index. This yeilds a first quadrant spectral sequence converging to $H^{*}\left(\mathbf{L}_{\pi}(G, m) ; G_{2 \phi_{2}}\right)$. Furthermore as a consequence of the relative injectivity of $C^{q}\left(\mathbf{K}\left(G_{1}, m\right) ; G_{2}\right)$ for $q>0$, as well as the existence of the section $s_{0}: \overline{\mathbf{W}}(\pi) \rightarrow \mathbf{L}_{\pi}\left(G_{1}, m\right)$, this spectral sequence collapses at the second term with

$$
E_{2}^{p, q}= \begin{cases}0 & \text { if } p>0 \text { and } q>0 \\ H_{\mathrm{ev}}^{q}\left(\mathbf{K}\left(G_{1}, m\right) ; G_{2}\right) & \text { if } p=0 \\ H^{p}\left(\mathbf{K}(\pi, 1) ; G_{2 \phi_{2}}\right) & \text { if } q=0\end{cases}
$$

Hence we have the following:

\section{Theorem 8.1.}

$$
H^{n}\left(\mathbf{L}_{\pi}\left(G_{1} ; m\right) ; G_{2 \phi_{2}}\right) \cong H_{\mathrm{ev}}^{n}\left(\mathbf{K}\left(G_{1}, m\right) ; G_{2}\right) \oplus H^{n}\left(\mathbf{K}(\pi, 1) ; G_{2 \phi_{2}}\right) .
$$

\section{References}

[1] G. Bredon, Equivariant cohomology theories, Lecture Notes in Math., vol. 34, Springer-Verlag, New York.

[2] S. Eilenberg, Homology of spaces with operators I, Trans. Am. Math. Soc., 61 (1947), 378-417.

[3] S. Eilenberg and J.C. Moore, Limits and spectral sequences, Topology, 1 (1961), 1-23.

[4] S. Gitler, Cohomology operations with local coefficients, Am. J. Math., 85 (1963), 156-188. 
[5] P.A. Grffiths and J.W. Morgan, Rational homotopy theory and differential forms, Progress in mathematics, vol. 16, Birkhauser, Boston, 1981.

[6] P.J. Hilton and U. Stammbach, A course in homological algebra, GTM 4, SpringerVerlag, New York, 1971.

[7] D.M. Kan, A combinatorial definition of homotopy groups, Ann. Math., 67 (1958), 282-312.

[8] J.P. May, Simplicial objects in algebraic topology, Univ. of Chicago Press, Chicago, Ill., 1982.

[9] J.F. McClendon, Obstruction theory in fiber spaces, Math. Zeitung, 120 (1971), $1-17$.

[10] P. Olum, Obstructions to extensions and homotopies, Ann. Math., 52 (1950), 1-50.

[11] J.P. Serre, Groupes d'homotopie et classes de groupes Abelienes, Ann. Math., 58(2) (1953).

[12] J. Siegel, Cohomology operations in local coefficient theory, Ill. J. Math., 15 (1971), 52-63.

[13] k-invariants and local coefficient theory, Proc. Am. Math. S., 29 (1971), 169-174.

Received March 30, 1993.

Phil Thurber

668 MCVEY Ave., \#70

Lake Oswego, OR 97034 